\title{
Generation of chaotic radiation in a driven traveling wave tube amplifier with time-delayed feedback
}

\author{
Chad Marchewka \\ Massachusetts Institute of Technology, 77 Massachusetts Avenue, Cambridge, Massachusetts 02139 \\ Paul Larsen \\ University of Wisconsin-Madison, 1415 Engineering Drive, Madison, Wisconsin 53706 \\ Sudeep Bhattacharjee \\ Indian Institute of Technology-Kanpur, Kanpur 208016, Uttar Pradesh, India \\ John Booske and Sean Sengele \\ University of Wisconsin-Madison, 1415 Engineering Drive, Madison, Wisconsin 53706 \\ Nikita Ryskin and Vladimir Titov \\ Saratov State University, Astrakhanskaya Street 83, Saratov 410026, Russia
}

(Received 21 September 2005; accepted 11 October 2005; published online 17 January 2006)

\begin{abstract}
The application of chaos in communications and radar offers new and interesting possibilities. This article describes investigations on the generation of chaos in a traveling wave tube (TWT) amplifier and the experimental parameters responsible for sustaining stable chaos. Chaos is generated in a TWT amplifier when it is made to operate in a highly nonlinear regime by recirculating a fraction of the TWT output power back to the input in a delayed feedback configuration. A driver wave provides a constant external force to the system making it behave like a forced nonlinear oscillator. The effects of the feedback bandwidth, intensity, and phase are described. The study illuminates the different transitions to chaos and the effect of parameters such as the frequency and intensity of the driver wave. The detuning frequency, i.e., difference frequency between the driver wave and the natural oscillation of the system, has been identified as being an important physical parameter for controlling evolution to chaos. Among the observed routes to chaos, besides the more common period doubling, a new route called loss of frequency locking occurs when the driving frequency is adjacent to a natural oscillation mode. The feedback bandwidth controls the nonlinear dynamics of the system, particularly the number of natural oscillation modes. A computational model has been developed to simulate the experiments and reasonably good agreement is obtained between them. Experiments are described that demonstrate the feasibility of chaotic communications using two TWTs, where one is operated as a driven chaotic oscillator and the other as a time-delayed, open-loop amplifier. @ 2006 American Institute of Physics. [DOI: 10.1063/1.2161170]
\end{abstract}

\section{INTRODUCTION}

Chaotic sources offer a new model for designing versatile, wide bandwidth rf sources for communications and radar applications. ${ }^{1-6}$ Here, one usually defines chaos as aperiodic (random-like) long-term behavior in a deterministic system that exhibits sensitive dependence on initial conditions. As opposed to conventional signal transmission involving modulation of pure harmonic carrier wave forms from stable generators, inherently unstable generators may offer more flexibility and functionality while operating under chaotic conditions. For example, with conventional harmonic carrier communications, power amplifiers are typically backed off from saturation in order to prevent harmonic and intermodulation distortion. This leads to reduced power efficiency and data rates. Communication or radar applications using chaos on the other hand would directly utilize nonlinear behavior in which the generator can operate in a saturated state and thus at maximum electronic efficiency. The covert nature of the transmitted message embedded in a chaotic wave form can potentially inhibit eavesdropping. By providing an efficient high power source of wideband radiation, chaotic transmitters offer exciting potential for highresolution $(\Delta x \sim 1 / \Delta k)$ ranging radar with low probability of detection and resistance to jamming. Digital communication schemes using wave forms from chaotic generators are predicted to offer high spectral efficiency, similar to spread spectrum encoding techniques.

As alluded to above, many of these attributes for covert, high-channel-density communications and covert, highresolution radar are also provided using spread spectrum coding methods. However, for long-distance communication links and long-range radar where high power transmitters are required, spread spectrum systems also require highly linear amplifiers. To reduce distortion, this generally requires operating amplifiers backed off from saturation, with a concomitant loss of efficiency. In contrast, as mentioned previously, operating a transmitter in a chaotic regime intentionally exploits its nonlinearities, allowing it to operate near saturation and closer to maximum efficiency.

To date, investigations of chaos-based communication systems have primarily emphasized optical lasers or low power solid-state devices (see, for example, Refs. 2 and 7-9 

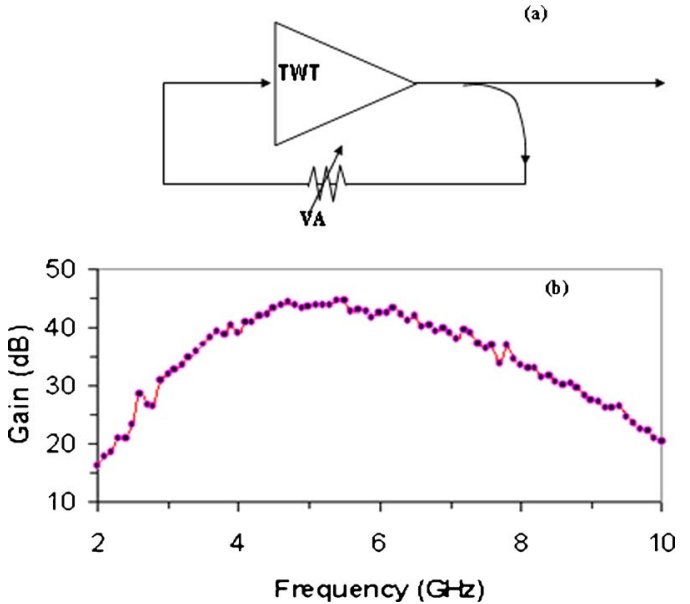

FIG. 1. (a) Open-loop oscillator configuration. VA is a variable attenuator to control the amount of feedback power. (b) Small signal gain vs frequency of the TWT amplifier.

and references cited therein). However, high power, efficiency, and "tether-free" requirements of radar or distance communications applications are difficult to meet in these devices. In contrast, microwave vacuum electronic transmitters are attractive because of their ability to generate high power at high efficiency in compact devices. There is also a system advantage in that a single transmitter can be used for both conventional carrier and noise (chaos) carrier operations.

The basic requirements for a chaotic communications system include (1) a pair of matched nonlinear oscillators (one for transmission and one for reception), (2) a method to embed and extract information from the chaotic carrier, and (3) identification of operating regimes exhibiting "robust" chaos. The third requirement addresses the fact that a reliable communications or radar transmitter cannot afford too much sensitivity to system settings, yet that same sensitivity to initial conditions that yields chaos can make it difficult to find or sustain chaos in the presence of signal modulations or inevitable drifts in system component properties.

This article describes the results of an investigation of chaotic wave form generation using traveling wave tubes (TWTs) with external delayed feedback. A few prior investigations as given in Refs. 10-12 identified some of the basic nonlinear dynamics of such a nonlinear oscillator. Recently, a theoretical study (see Ref. 13) presented results supporting the feasibility of one method for controlled chaos for encode and decode purposes. In this article, we describe results of experimental and computational studies of driven TWT oscillators, where an injected monochromatic wave is shown to be a highly effective means to induce and control chaotic dynamics.

The article has been arranged as follows. Section II describes the experimental configuration, methods, and results. Section III describes the numerical model and the simulation results. Section IV describes results of an experimental examination of chaotic communications using two TWTs. A summary and conclusions are provided in Sec. V.

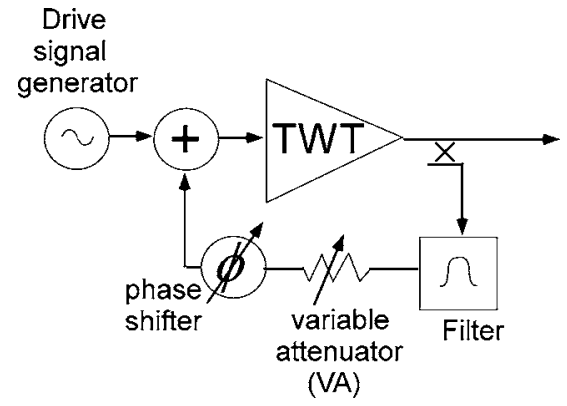

FIG. 2. Schematic of a more advanced experimental setup, including a drive signal generator and means to control the feedback signal, including a variable attenuator, phase shifter, and bandpass filter.

\section{EXPERIMENTAL CONFIGURATION AND RESULTS}

The basic experimental configuration consists of a TWT with external recirculated feedback as shown in Fig. 1. For the experiments described in this paper, the TWT was a Varian VTC-6067A1 with positive gain over a relatively large bandwidth from approximately $1-12 \mathrm{GHz}$. The maximum small signal gain was approximately $45 \mathrm{~dB}$ and the $-3 \mathrm{~dB}$ bandwidth ranged between 4.2-6.5 GHz. Exploratory experiments quickly identified, however, that finding and studying chaos with this configuration were very challenging for two reasons.

First, the available variable attenuator in the feedback leg was limited to discrete adjustments of $\pm 1 \mathrm{~dB}$ increments. It was hypothesized that this level of adjustment control was not precise enough to move the system in and out of chaos. This hypothesis was eventually confirmed by simulations, described later in the paper. As an alternative, the configuration was modified by adding a harmonic, monochromatic drive signal generator, as indicated in Fig. 2. Using a commercial signal generator (Agilent 83623B), the power and
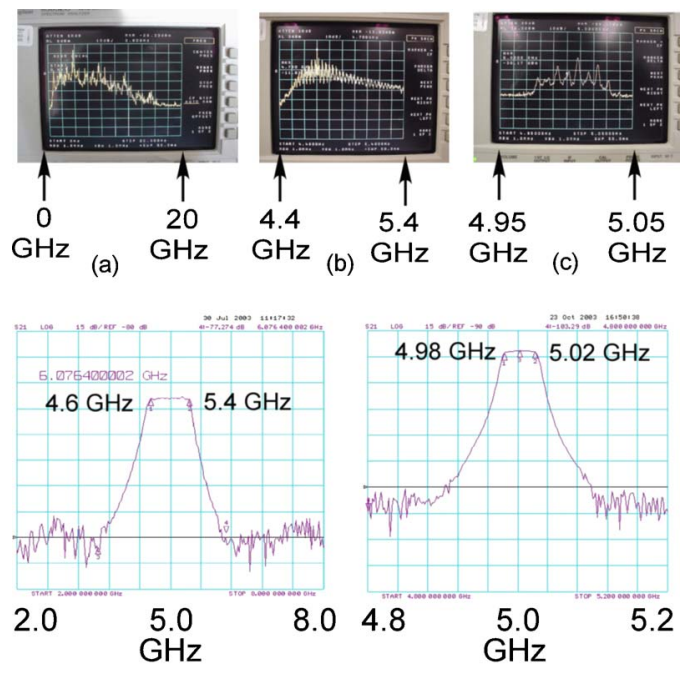

(d)

(e)

FIG. 3. Power spectra of chaotic wave forms with (a) no filter ( $2 \mathrm{GHz} / \mathrm{div})$, (b) $800 \mathrm{MHz}$ bandpass filter (100 MHz/div), and (c) $40 \mathrm{MHz}$ bandpass filter $(10 \mathrm{MHz} / \mathrm{div})$ in the feedback circuit. In all three spectra, the vertical scale represents $10 \mathrm{~dB} /$ div. Also shown are frequency response curves for the (d) $800 \mathrm{MHz}$ and (e) $40 \mathrm{MHz}$ bandpass filters. 

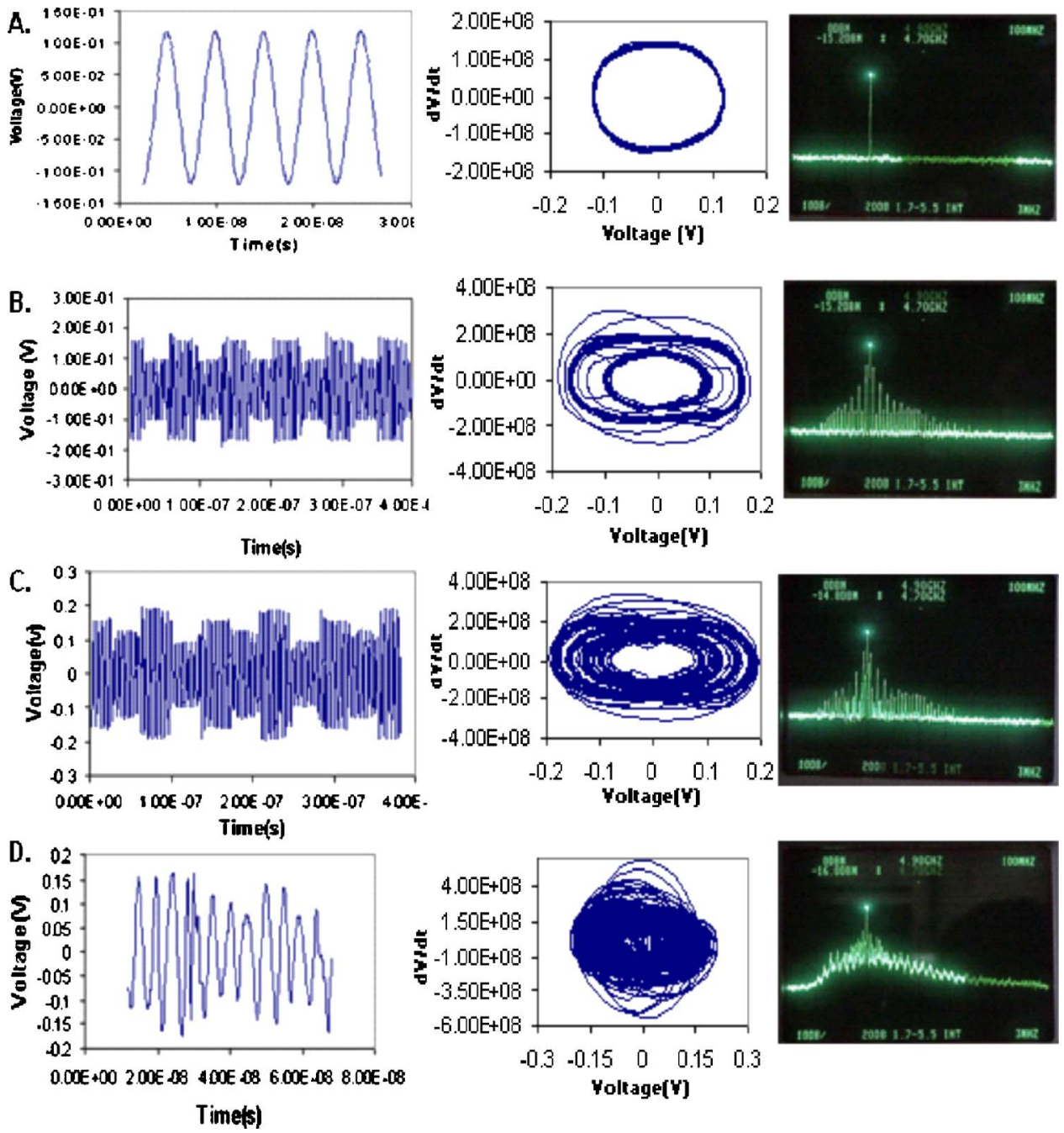

Yoltage[Y]

the frequency of this drive signal were variable with extremely precise control. Most of the detailed experimentation involved operating the drive signal generator near the point of maximum small signal gain, i.e., near $\sim 5 \mathrm{GHz}$, although several experiments (not described in this paper) indicated that most of the basic phenomena reported in this paper were observable for drive signal frequencies chosen throughout much of the TWT's positive gain bandwidth. Also, as indicated in Fig. 2, a phase shifter was added to the feedback leg, for an additional source of precise parameter adjustment. This will be further discussed shortly.

The second challenge arose as a result of the TWT's very large gain bandwidth. Experimental studies of chaos benefit from capture of time-domain wave forms, along with frequency-domain spectra. Preliminary experiments using spectral analysis revealed a transition to chaos during which the power spectrum shifted from multiple simultaneous discrete frequencies to broadband "noise" while tuning various experimental parameters (such as the phase of the feedback leg). The difficulty was encountered when results such as those in Fig. 3(a) were observed. In this case, the bandwidth of the signal is well in excess of $10 \mathrm{GHz}$. Capturing instantaneous time-domain wave forms of complex chaotic signals with this much bandwidth was beyond the capabilities of the available digital data acquisition instrumentation. Conse- quently, a bandpass filter was added to the feedback circuit to limit the bandwidth of the signals.

Experiments were conducted with two candidate feedback filters, one of them having an $800 \mathrm{MHz}$ passband [Fig. $3(\mathrm{~d})$ ] and a second one having a $40 \mathrm{MHz}$ passband [Fig. $3(\mathrm{e})]$. Figure 3(b) is an example of the spectrum for a chaotic state observed with the $800 \mathrm{GHz}$ passband filter in the feedback leg, and Fig. 3(c) is a spectrum of a chaotic state observed with the $40 \mathrm{MHz}$ filter in the feedback leg. These results confirmed that the feedback bandpass filter successfully limited the signal's bandwidth to the filter's bandwidth, i.e., 800 and $40 \mathrm{MHz}$ in Figs. 3(b) and 3(c), respectively.

Other differences were observed in the system dynamics when using the two different bandpass feedback filters. For these observations, the output signal was subjected to both frequency and time-domain analyses. The frequency-domain analysis consisted of conventional spectrum analyzer measurements, while the time-domain analysis was accomplished by down converting the signal using a mixer (ANZAC MDC-171), an auxiliary synthesized local oscillator signal, and a $6 \mathrm{GHz}$ bandwidth digital capture oscilloscope (Agilent Infinium 54855A DSO). In both cases, a period-doubling-type transition to chaos was observed when the phase shifter in the feedback leg was varied, holding all other experimental variables constant. Figure 4 shows (down 
(a)

(b)

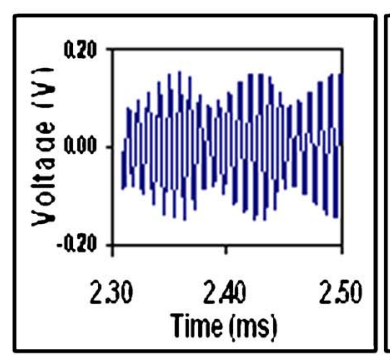

(c)

(d)
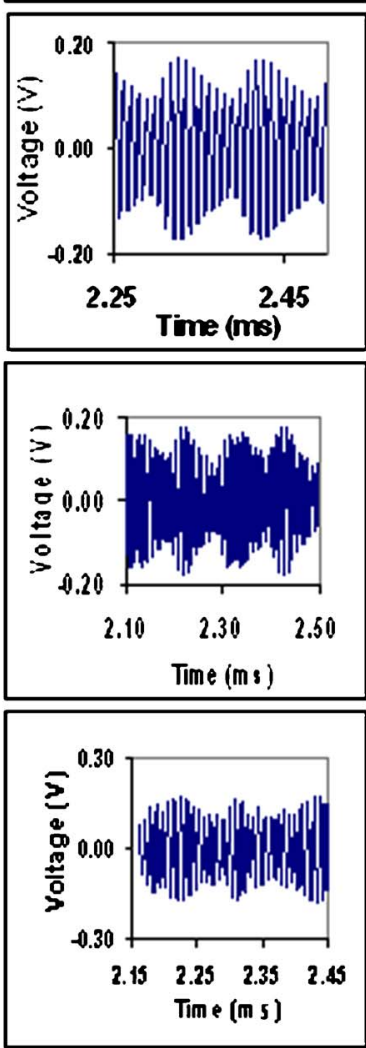
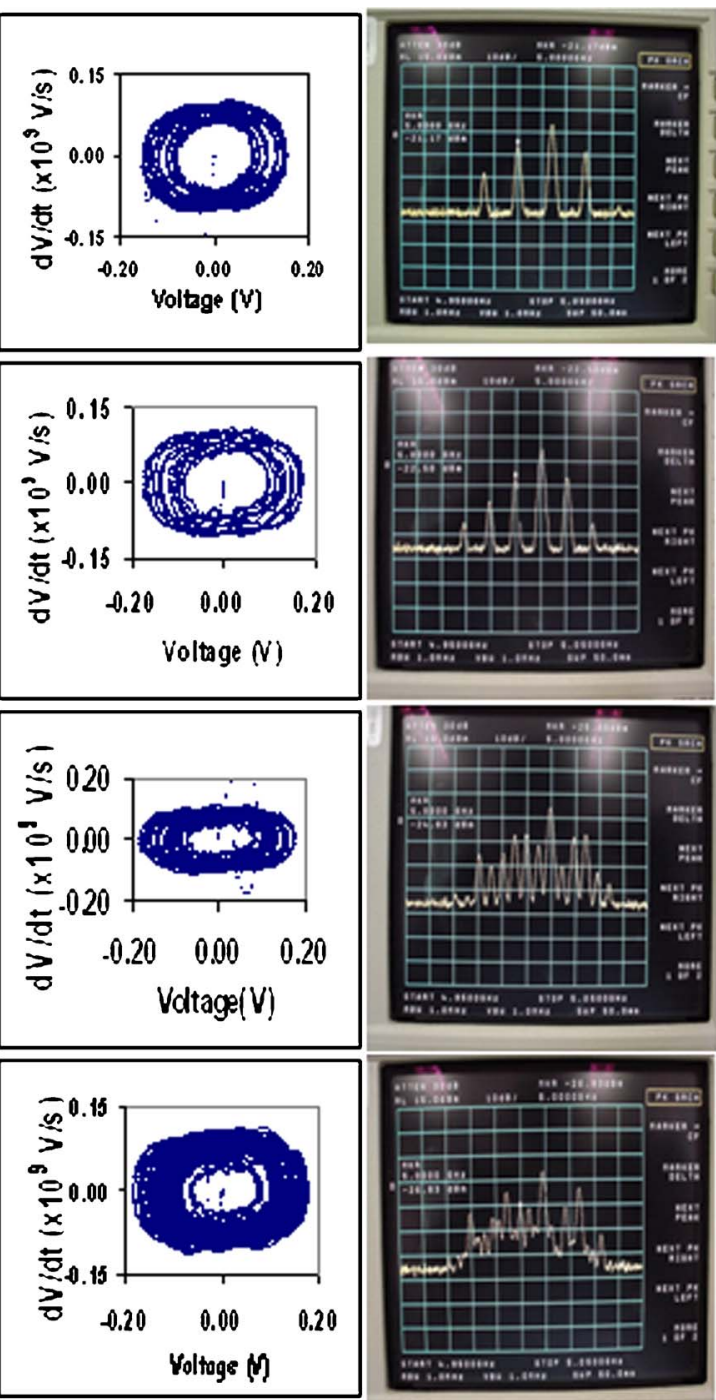

FIG. 5. Phase-space portrait, time series, and spectral images obtained in the case of a narrow band $(40 \mathrm{MHz})$ filter. The data represent adjustment of the feedback leg's phase shifter to (a) $10.2^{\circ}$, (b) $30.2^{\circ}$, (c) $33.2^{\circ}$, and (d) $35.9^{\circ}$ at a constant drive frequency and power of $5 \mathrm{GHz}$ and $11 \mathrm{dBm}$, respectively, and feedback leg attenuator level of $-3 \mathrm{~dB}$. converted) time-domain wave forms $V(t)$, time-averaged spectra, and phase-space plots of the wave form obtained by plotting $V(t)$ versus its derivative $d V(t) / d t$. Figure 5 shows a similar set of data obtained by replacing the $800 \mathrm{MHz}$ bandwidth filter with the $40 \mathrm{MHz}$ bandwidth filter. The $6 \mathrm{GHz}$ oscilloscope added a larger than acceptable high-frequency internal jitter to the data. In order to minimize this internal oscilloscope jitter, the data were processed using a MATLAB low pass $1.5 \mathrm{GHz}$ Butterworth software filter in addition to the low pass hardware filter shown in Fig. 2. Both experiments indicate a transition from single frequency to amplitude modulation and ultimately to chaos through period doubling of the amplitude modulation envelope. However, while the experiment using the narrowband $40 \mathrm{MHz}$ filter exhibited a sinusoidal envelope modulation, the experiment using the $800 \mathrm{MHz}$ bandwidth feedback filter manifested a squarewave envelope modulation. These observations will be further discussed in Sec. III describing the numerical simulations.

After reviewing the preliminary experimental results, a final set of configuration choices was made. As discussed in Sec. III and confirmed by experiments, it was established that the effect of the phase shifter in the feedback leg was to modify the resonant eigenfrequencies of the ring-loop cavity defined by the TWT and the feedback leg. Furthermore, it was experimentally confirmed that what was fundamentally most important was the value of the relative frequency difference between the drive signal and any of the resonant eigenfrequencies, rather than the absolute value of either the drive frequency or any of the resonant eigenfrequencies.

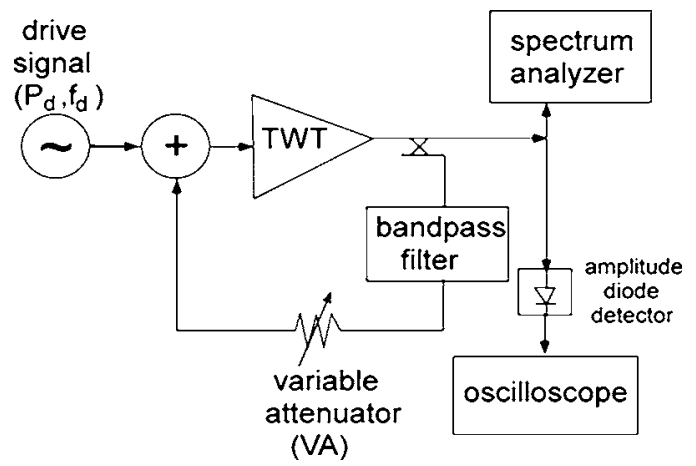

FIG. 6. Schematic of the final experimental setup. Notice the removal of the phase shifter and the addition of an amplitude diode detector for obtaining the envelope of the waves. 

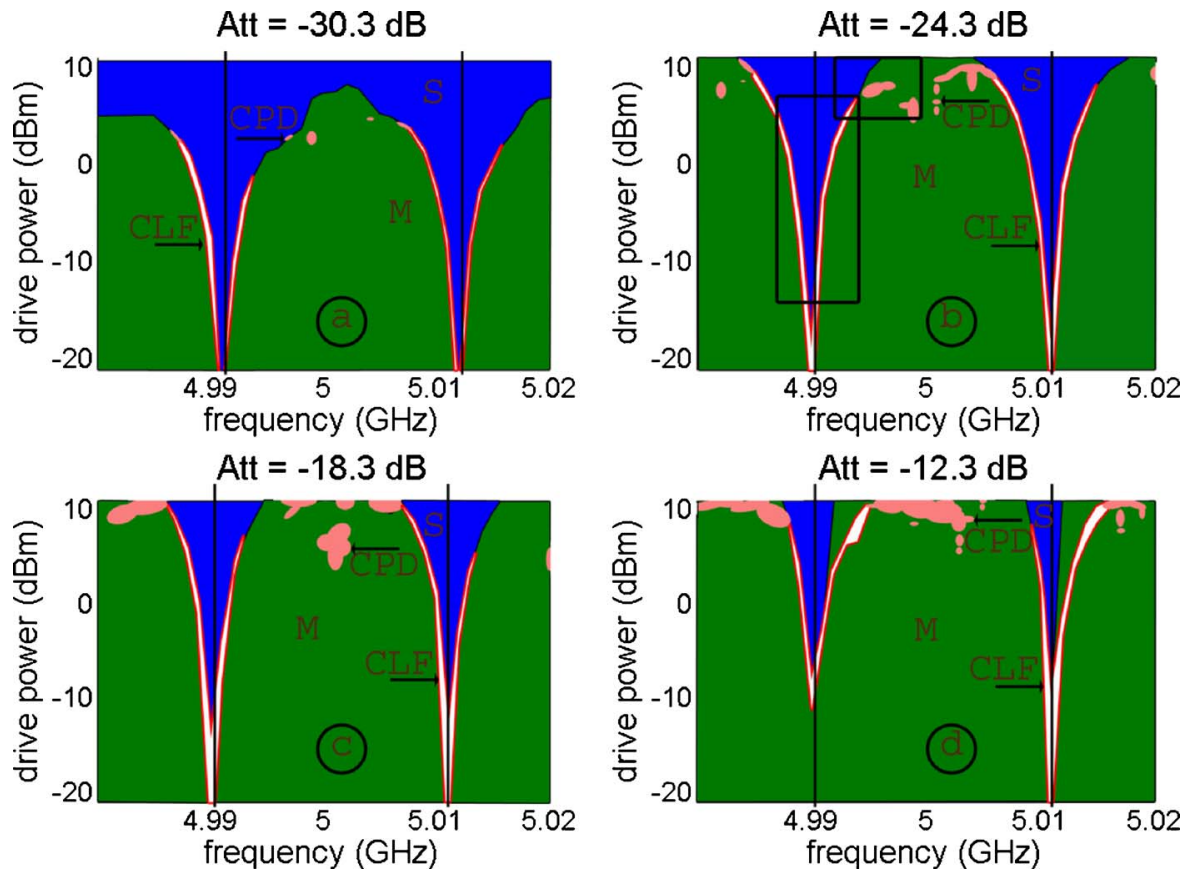

FIG. 7. Arnol'd maps for drive power vs drive frequency at four different levels of feedback attenuation, (a) $-30.3 \mathrm{~dB},(\mathrm{~b})-24.3 \mathrm{~dB},(\mathrm{c})-18.3 \mathrm{~dB}$, and (d) $-12.3 \mathrm{~dB}$. "Att" is the total feedback circuit attenuation (measured), and "drive power" is the cw power at the input to the TWT (after losses in the cable and combiner). The various color codes are green: multifrequency $(M)$ and blue: single frequency (the drive frequency) $(S)$. The pink area is the chaos, solid pink (CPD) is period-doubling chaos, and white surrounded by pink (CLF) is the loss-of-frequency-locking chaos. The black vertical lines are where the natural self-oscillation resonances exist. The boxes in (b) are where the detailed maps of Fig. 8 are located.
Consequently, it was confirmed that the phase shifter in the feedback leg was redundant, and that control of dynamics through the frequency parameter could be controlled by variation of the drive frequency alone. At the same time, it was determined from these preliminary experiments and theoretical analyses (discussed in Sec. III) that the basic nonlinear dynamics of this chaotic TWT oscillator involved nonlinear couplings of the resonant eigenfrequencies of the ringloop circuit and the drive signal. The frequency spacing between these eigenfrequencies was determined, in part, by the electrical length of the feedback leg as that affected the round-trip delay time. Therefore, it was decided to use the narrow $40 \mathrm{MHz}$ bandwidth filter and minimize the time delay of the feedback leg, in order to limit the number of simultaneous eigenfrequencies to $\sim 2-3$ and thereby simplify the dynamics for theoretical and experimental studies. In addition, an amplitude diode detector was inserted before the oscilloscope input. This was necessary to capture the $\sim 5 \mathrm{GHz}$ signal without a mixer in order to produce phase maps that more clearly characterize period doubling and other chaotic behavior. The final experimental configuration therefore resembled that of Fig. 6.

A detailed study was conducted on the nonlinear dynamics of the configuration of Fig. 6, as a function of variations in two control variables: the power level and the frequency of the drive signal. The $40 \mathrm{MHz}$ bandpass filter had an adjustable center frequency. The first measurement was to remove the drive signal, reduce the feedback attenuation, allow the system to operate as a free-running oscillator, adjust the center frequency of the bandpass filter, and record the values of several of the resonant ring-loop eigenmodes. In particular, it was observed that two natural resonances near the peak of the TWT's small signal gain occurred at 4.99 and $5.012 \mathrm{GHz}$. Subsequently, it was decided to conduct all measurements in the vicinity of these two resonances.

Measurements were taken by varying the drive fre- quency at constant drive signal power and by varying the drive signal power at constant drive frequency. Arnol'd maps were constructed from these data, and several examples are illustrated in Fig. 7 for four level settings of the variable feedback attenuator. A dominant phenomenon of a driven oscillator is mode locking. The areas in parameter space where mode locking occurs are usually referred to as Arnol'd tongues after the discoverer. They are bordered by saddlenode bifurcations. The areas enclosed by the saddle-node bifurcation curves may not only contain mode locked oscillations but also period-doubling cascades and chaos. The maps of Figs. 7 and 8 depict regions of single frequency, multifrequency, and chaotic operation. The green regions depicted by the letter " $M$ " in the figures exhibited stable, multifrequency behavior either periodic or quasiperiodic in which the power spectra exhibited stationary discrete spectral lines. The blue regions depicted by the letter " $S$ " at high drive powers exhibited stable, single-frequency (i.e., the drive frequency) behavior. Chaos was observed in the pink regions and white-surrounded-by-pink regions at the boundaries between discrete-multifrequency and stable singlefrequency dynamics. Expanded views with additional detail are shown in Fig. 8 from the case where the feedback attenuation was $-24.3 \mathrm{~dB}$. These figures show that a fine structure exists to the chaotic regions. The pink chaotic regions (depicted by the letters "CPD") such as that of Fig. 7(b) occurring midway between two natural resonances are islands surrounded by discrete multitone dynamics. The island edges tended to be a combination of discrete multitone and weak chaos, whereas robust chaos existed in the center of the islands. In contrast, the white-surrounded-by-pink regions of chaos depicted by the letters "CLF" such as that of Fig. 7(a) occurring near one of the natural resonance frequencies were typically continuous, narrow bands bordering the edges of the Arnol'd tongues.

Two distinctly different types of chaos were observed, 

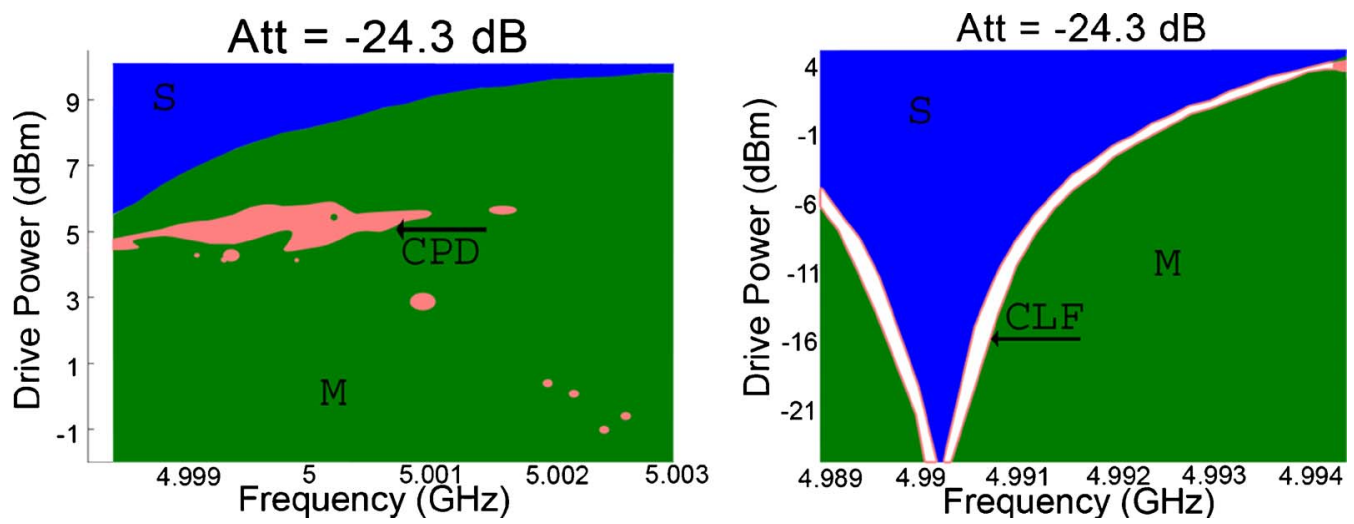

FIG. 8. Detailed Arnol'd maps corresponding to the region shown in boxes in Fig. 7(b).

distinguished by CPD and CLF regions of Figs. 7 and 8 . When the drive frequency was set to a value approximately halfway between two of the natural resonances, a perioddoubling type of chaos was observed. In contrast, when the drive frequency was close to one of the natural oscillation frequencies, a different type of chaos was observed, which we have labeled as "loss-of-frequency-locking" chaos.

Figure 9 illustrates time-domain envelope wave forms and time-averaged spectra of the case of period-doubling chaos. The drive signal power was held constant (at $5 \mathrm{dBm}$ ) and the drive frequency was varied, i.e., moving along a horizontal trajectory in Fig. 7(b). As the drive frequency was increased, the characteristics of the wave forms changed from a stable single-frequency to period-two-modulated oscillation, then period-four-modulated, period-eightmodulated oscillations, and finally chaos.

Figure 10 illustrates the case of loss-of-frequencylocking chaos. In this case, as the drive frequency was varied in a direction approaching one of the natural oscillation frequencies, the system evolved through a region of periodic modulation. As the drive frequency was adjusted to more closely equal one of the natural oscillation frequencies, the modulation frequency correspondingly decreased in a smooth, continuous fashion. Correspondingly, the spectrum of discrete frequencies became more densely packed while the intertone spacing continuously decreased until one reached a critical frequency difference. At this critical frequency difference, the discrete spectrum became continuous and the time-domain wave form adopted an aperiodic chaotic signature. Moving the drive frequency even closer to the natural oscillation frequency resulted in the disappearance of the chaos and the appearance of stable, single-frequency, unmodulated oscillations.

In another experiment the power of the drive signal was varied at a constant drive frequency. Figure 11 shows the time-domain wave forms and the phase-space plots for the period-doubling chaos when the injected drive power was varied from -10 to $17.5 \mathrm{dBm}$ at a constant drive frequency of $5.002 \mathrm{GHz}$, and Fig. 12 shows a similar experiment with the drive frequency set at $4.989 \mathrm{GHz}$ for the loss-offrequency-locking chaos.
The time-domain wave forms and phase-space plots for the period-doubling chaos (Fig. 11) clearly indicate the transitions from period one to period eight and finally into chaos. In the case of loss-of-frequency-locking chaos (Fig. 12), the drive frequency was kept fixed at $4.989 \mathrm{GHz}$ (close to a natural resonant frequency), and as injected power was increased, the wave amplitude became periodically modulated. The modulation frequency was observed to decrease with increase in drive power and then finally transitioned to chaotic wave forms. Chaos disappeared as the drive power was further increased and there was perfect frequency locking to the drive frequency.

\section{NUMERICAL MODEL AND SIMULATIONS}

\section{A. General considerations}

Nonstationary simulation of a TWT oscillator is a challenging task. Several published studies have been based on the slowly varying envelope approximation (SVEA) as given in Refs. 14-19, and the dynamics of the system are well studied from this point of view. SVEA theory is valid only when the radiation spectrum is relatively narrow and it typically does not take into account dispersion of group velocity and coupling impedance. ${ }^{18,19}$ For a broadband helix TWT the most natural and comprehensive approach involves the application of particle in cell (PIC) codes such as MAGIC (see, for example, Ref. 20) or MAFIA (see, for example, Ref. 21). However, studying chaotic processes requires calculations of long-time evolution. This presents a challenge for full-scale PIC simulation because of time requirement constraints, and only qualitative results for a device with substantially reduced length have been obtained until now. ${ }^{22}$

On the other hand, if a passband filter is included in the feedback section of a broadband TWT regenerative oscillator circuit, significant simplification of the numerical model can be achieved and an SVEA model can be employed. Let us express the high-frequency field as

$$
E(x, t)=\operatorname{Re}\left[\mathrm{E}(x, t) e^{i\left(\omega_{0} t-\beta_{0} x\right)}\right],
$$

where $\omega_{0}$ is the central frequency of the filter passband, and 


\section{time dependent amplitude}
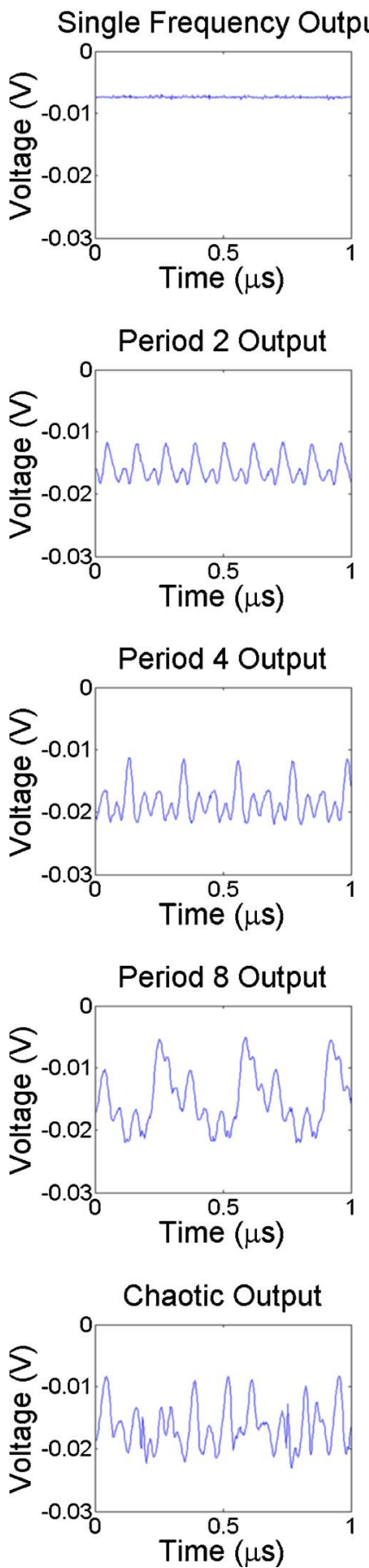

\section{spectrum}

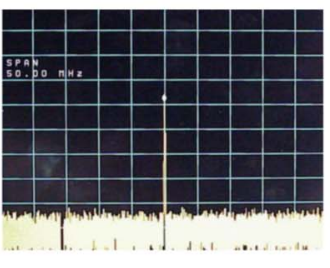

\section{stable oscillation single frequency (synchronization)}

\author{
periodic \\ modulation
}

\author{
period-two
} modulation

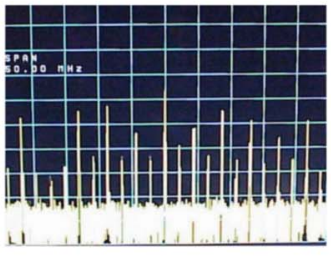

\section{period-four modulation}

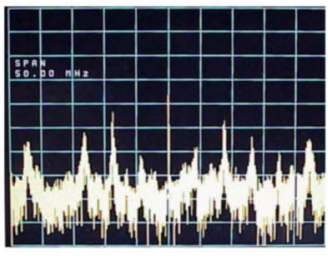

\section{chaos}

FIG. 9. Time-domain wave forms and spectral images of period-doubling chaos. The drive frequency was varied over the range $4.97-5.04 \mathrm{GHz}$ at a constant drive power of $5 \mathrm{dBm}$.

$E$ is the complex slowly varying envelope. In a TWT amplifier perturbations are transferred along the tube by two ways, namely, by the high-frequency wave and the electron beam. Characteristic velocities of those two processes are $v_{g}$ (the group velocity) and $v_{0}$ (the dc electron velocity), respectively. Thus, the output signal at any moment of time $\mathrm{E}_{\text {out }}(t)$ depends on the input signal $\mathrm{E}_{\mathrm{in}}\left[t \in\left(t-l / v_{0} ; t-l / v_{g}\right)\right]$ within a time interval of length $t_{s}=l / v_{g}-l / v_{0}$ called the slippage time [see discussions in Refs. 16 and 14 for TWTs and backward wave oscillators (BWOs), respectively]. Here $l$ is the length of the tube interaction space. However, if the spectrum bandwidth $\Delta \omega$, which is determined by the filter bandwidth, is small enough so that $\Delta \omega t_{s} \ll 1$, the slippage is negligible and $E_{\text {out }}$ can be supposed as an instantaneous function of $E_{i n}$ : 


\section{time dependent amplitude}
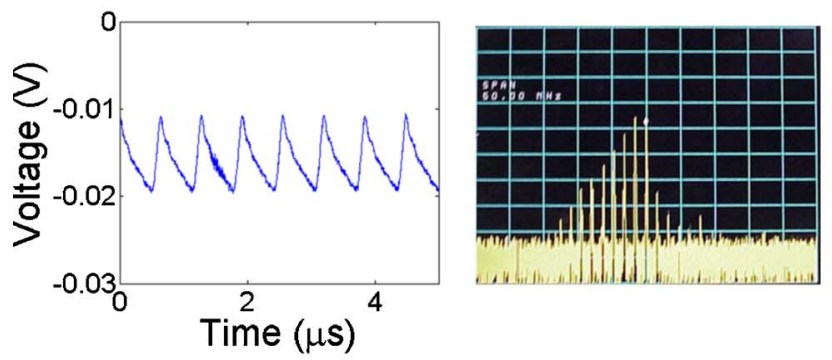

\section{periodic modulation}
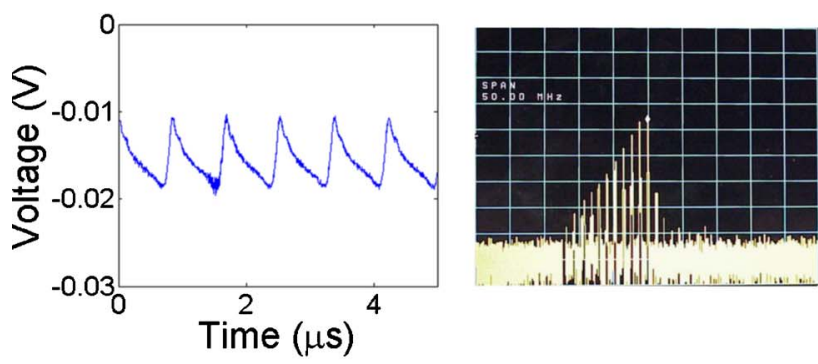

\section{decreasing} difference between drive and natural
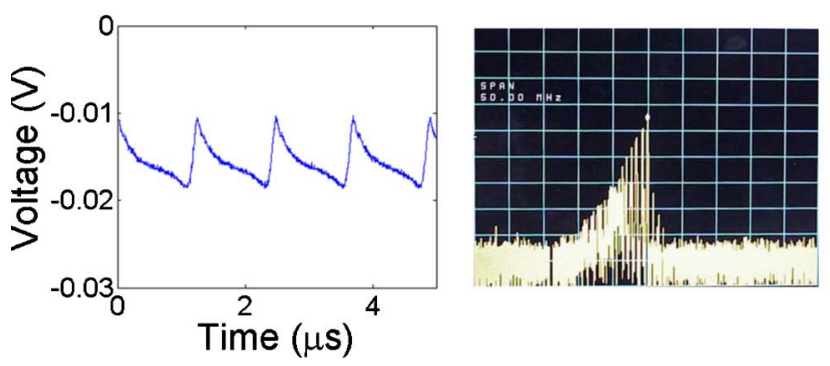
oscillation frequencies

FIG. 10. Time-domain wave forms and spectral images of loss-offrequency-locking chaos. The difference frequency between the drive and the natural oscillation frequencies was varied at a constant drive power of $5 \mathrm{dBm}$.
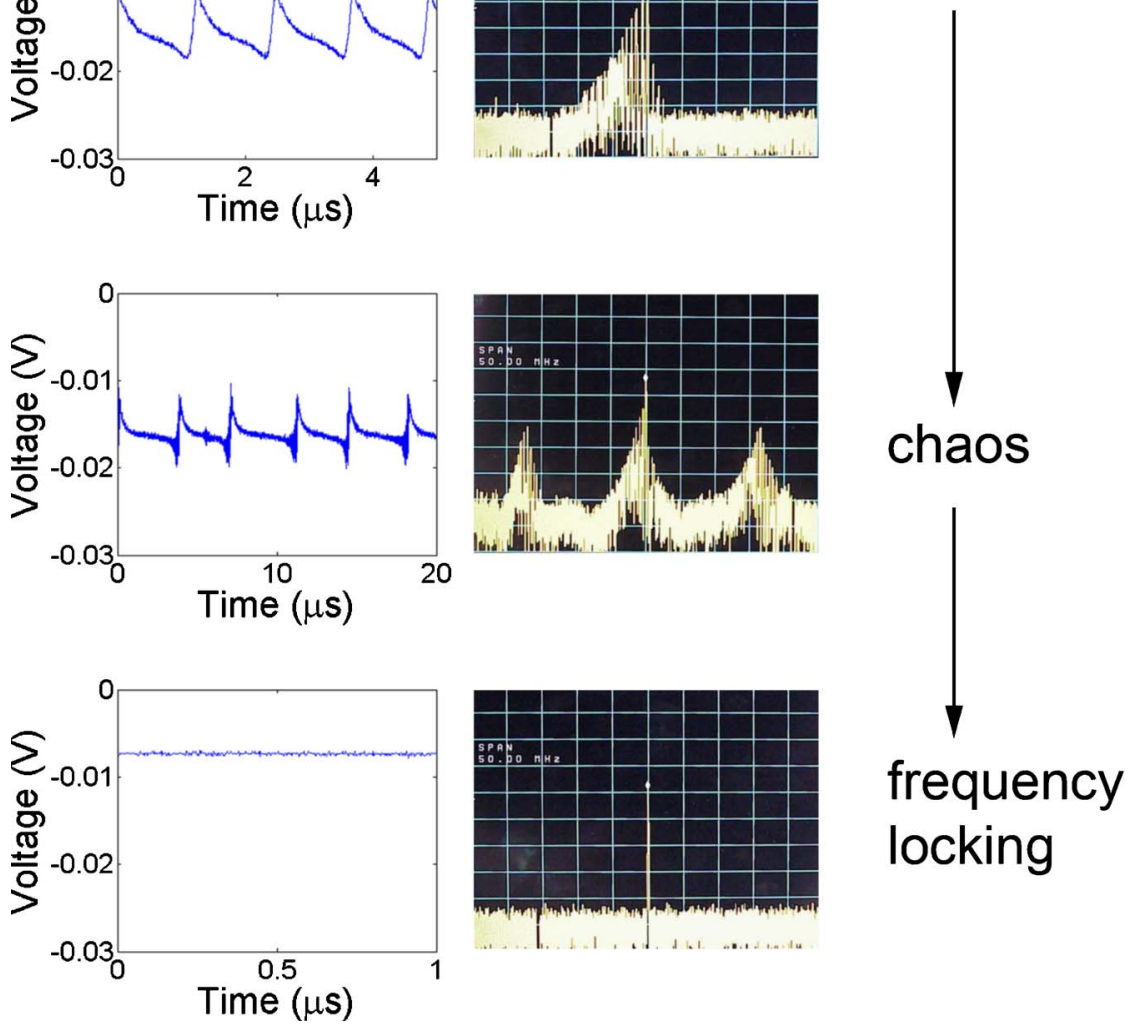

\section{frequency locking}

$$
\begin{aligned}
\mathrm{E}_{\text {out }}(t)= & \frac{G\left[\left|\mathrm{E}_{\text {in }}\left(t-l / v_{0}\right)\right|\right] \mathrm{E}_{\text {in }}\left(t-l / v_{0}\right)}{\left|\mathrm{E}_{\text {in }}\left(t-l / v_{0}\right)\right|} \\
& \times \exp \left(i \Phi\left[\left|\mathrm{E}_{\text {in }}\left(t-l / v_{0}\right)\right|\right]-i \beta_{0} l\right),
\end{aligned}
$$

where the nonlinear amplitude transfer function $G$ and the nonlinear phase transfer function $\Phi$ describe the AM/AM and AM/PM transfer curves, respectively. These functions can be either calculated or measured experimentally. Note 

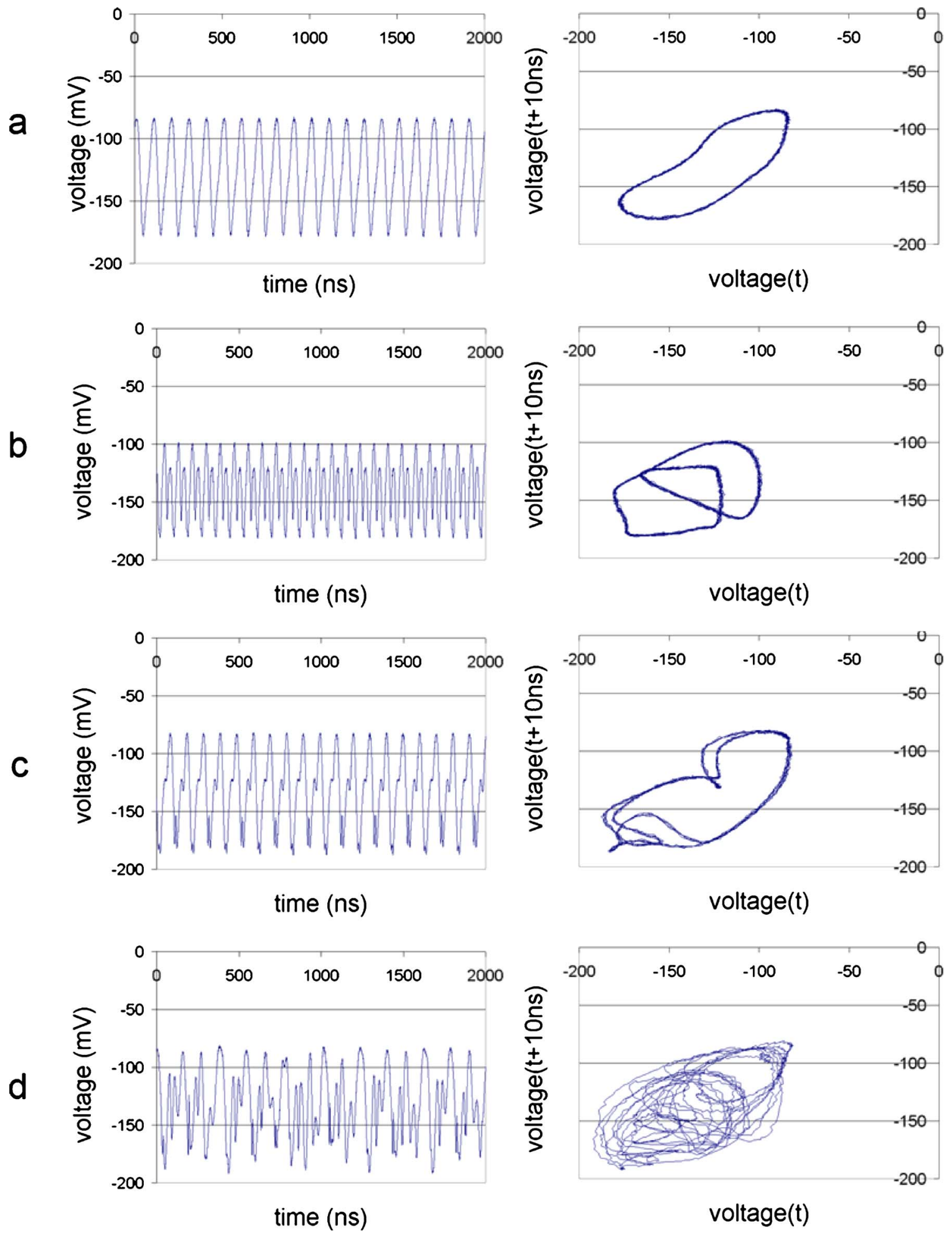

FIG. 11. Time-domain wave forms and phase-space plots of period-doubling chaos. The drive power was varied over the range $-10-17.5 \mathrm{dBm}$ at a constant drive frequency of $5.002 \mathrm{GHz}$.

that expressing $\mathrm{E}_{\mathrm{out}}$ as (2) assumes that the TWT gain can be considered as frequency independent within the filter passband.

In the particular case of the TWT oscillator configuration presented in Fig. 2 with the filter response (Fig. 3) represented by a Lorenz-shaped frequency dependence, one can obtain the following delay-differential equation for the wave envelope:

$$
\begin{aligned}
\frac{1}{\gamma} \dot{F}(\tau)+F(\tau)= & \frac{G[\rho|F(\tau-\delta)|] F(\tau-\delta)}{|F(\tau-\delta)|} \\
& \times \exp [i \Phi(\rho|F(\tau-\delta)|)+\psi] .
\end{aligned}
$$

Here $F=\mathrm{E} /\left(2 \beta_{0} V_{0} C^{2}\right)$ is the normalized signal envelope in the feedback loop, $V_{0}$ is the dc beam voltage, $C$ is the Pierce gain parameter, $\tau=v_{0} t / l$ is normalized time, $\delta=1+v_{0} \Delta t / l$ is 

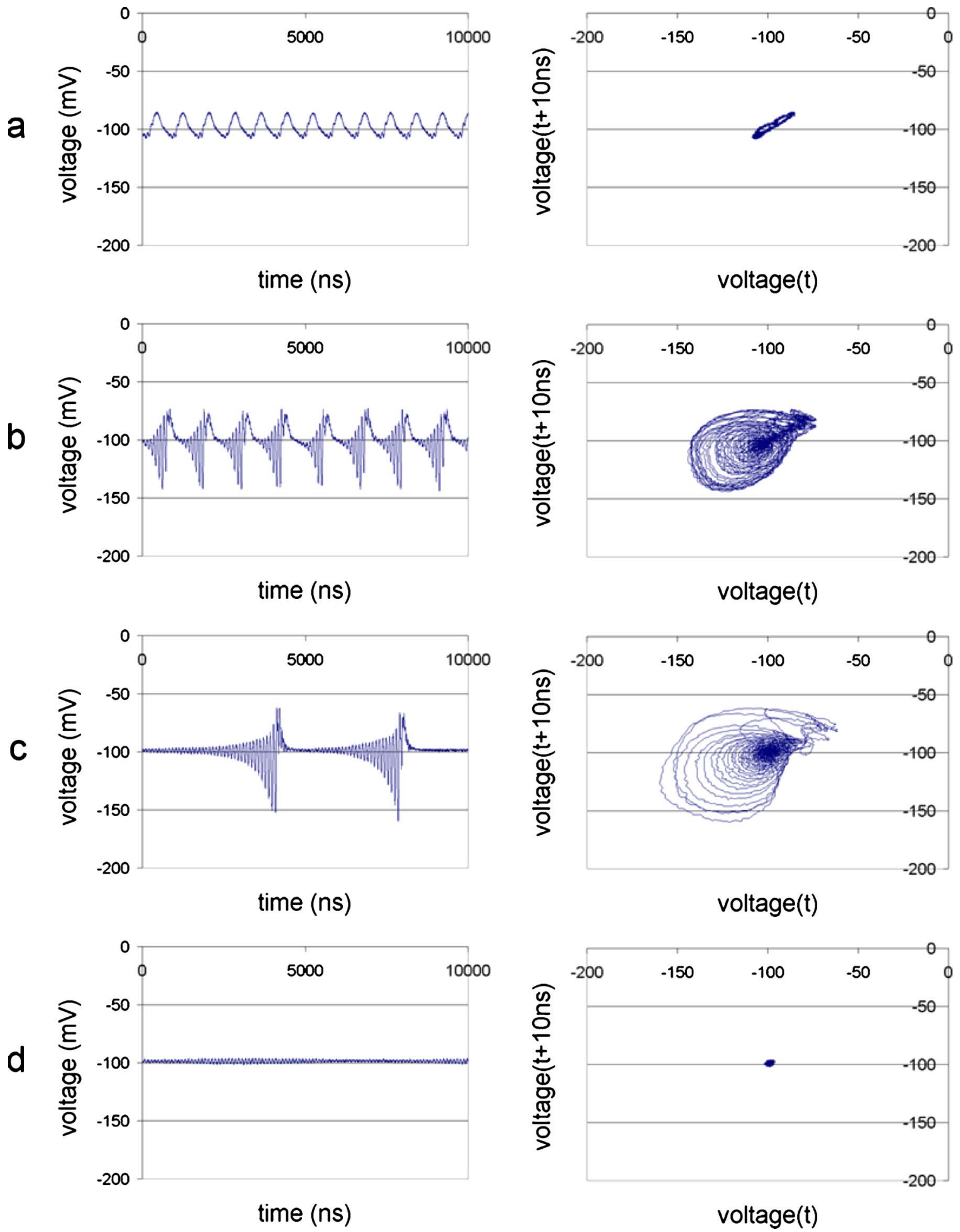

FIG. 12. Time-domain wave forms and phase-space plots of loss-of-frequency-locking chaos. The drive power was varied over the range $-10-17.5 \mathrm{dBm}$ at a constant drive frequency of $4.989 \mathrm{GHz}$.

a normalized delay time, $\Delta t$ is the time of propagation through the feedback circuit, $\rho$ determines the amount of feedback, $\psi=\Psi-\omega_{0} \Delta t-\beta_{0} l$, and $\Psi$ is a phase shift introduced by a phase shifter (see Fig. 2). The filter bandwidth is determined by the parameter $\gamma=\theta_{0} / 2 Q$, where $\theta_{0}=\omega_{0} l / v_{0}$ is the electron transit angle in the interaction space, and $Q$ is the filter $Q$ factor. In this model the TWT is represented as a "black box" completely specified by its AM/AM and
$\mathrm{AM} / \mathrm{PM}$ transfer curves, recorded at the carrier frequency equal to the central frequency of the filter. For example, a similar approach to simulations of chaos in a TWT oscillator was applied in Ref. 13. However, one important difference should be pointed out. In Ref. 13 it is supposed that finite bandwidth of the TWT amplifier acts as a kind of filter. Numerous results as given in Refs. 15-19 show that in this case the self-modulation mechanism and transition to chaos sce- 
nario are quite different from those predicted by the simple model (3) and are determined by the change of the TWT gain-frequency response shape in nonlinear regimes. The equation (3) is valid only for the TWT with a bandpass filter actually included in the feedback loop.

The cold structure formed by the TWT's helix slow wave structure and the feedback circuit can be treated as a ring-loop resonator. It is straightforward to show that the eigenfrequencies of the resonator are given by the formula

$$
\Omega_{n}=\frac{2 \pi n+\psi}{\delta}, \quad n=0, \pm 1, \pm 2, \ldots .
$$

The idealized system possesses an infinite set of spectrally equidistant, cold eigenmodes. The parameter $\delta$ is responsible not only for the feedback time itself, but for the relation of the amplifier bandwidth to the frequency separation between neighboring modes, i.e., with larger feedback time $\delta$, the eigenmodes are more closely spaced, and more of them will lie within the amplifier's band of positive gain. As mentioned in a previous section, (4) reveals that the eigenmode frequencies can be altered either by adjusting the lumped phase parameter $\psi$ or by changing the physical length of the feedback leg to change the feedback time $\delta$.

Nonlinear dynamics of delayed feedback systems similar to (1) have been examined in several works (see, for example, Ref. 23 and references therein). The typical observation is that a gradual increase of either gain or the amount of feedback leads to self-modulation and consequently perioddoubling transition to chaos. For delayed feedback TWTs, there exist two basic mechanisms of self-modulation, known as the amplitude and phase (or frequency) mechanisms as given in Refs. 15, 16, 24, and 25. The former can be attributed to the amplitude nonlinearity, while the latter to the phase nonlinearity. For the TWT with a narrowband filter in the feedback leg, the amplitude mechanism dominates. In this case, the self-modulation is caused by the presence of a steep negative slope in the AM/AM curve. ${ }^{10,15,16}$ The qualitative explanation to the amplitude mechanism is as follows. Consider the stationary regime when the TWT gain is close to or equal to the feedback attenuation. If at some moment of time due to fluctuations the input power exceeds the stationary value, the instantaneous output power will decrease. As a result of a decrease in the output power, after passing through the feedback circuit, which takes a time $\delta$, the signal amplitude will drop below the stationary value. Therefore it will be amplified again and, in turn, will exceed the stationary output level. Depending on the steepness of the negative slope of the AM/AM curve, such oscillations of power will decay or grow in time. In the first case a stable, periodic state is realized. In the second case growing amplitude perturbations give rise to periodic self-modulation with period about $2 \delta$ instead of the single-frequency oscillations (for a more rigorous derivation see Ref. 24). Subsequent increasing of either the amount of feedback or the TWT gain result in a period-doubling transition to chaos. ${ }^{10,15,16,24,26}$

For a TWT with a narrowband filter in the feedback leg, therefore, the role of the phase nonlinearity becomes subsidiary and basically leads to a nonlinear frequency shift leading to more uniform spectrum in the chaotic regime. It has been observed, for example, that in a nonstationary regime with strong amplitude modulation, the effect of the AM/PM nonlinearity causes "wobbling" of the basic frequencies and thus broadening of discrete spectral lines. ${ }^{17}$

In contrast, when there is no filter in the feedback loop and the TWT gain bandwidth is very broad, phase nonlinearity plays the primary role as given in Refs. 15-19. Selfmodulation arises via a phase or frequency mechanism caused by saddle-shaped deformation of the amplifier gainfrequency response near the dominant frequency in the strongly nonlinear regime. The corresponding onset to chaos generally occurs through the quasiperiodic route or through intermittency. ${ }^{18}$

\section{B. Simulations without an external driving wave}

For simulating the present experiment, the following analytical approximations of the amplitude and phase nonlinearities were used:

$$
\begin{aligned}
G(|F|)= & 102.119|F|\left(0.45 \exp \left(-124.82|F|^{2}\right)\right. \\
& +0.3 \exp (-15.881|F|))
\end{aligned}
$$

The approximations (5) and (6) (see Fig. 13) were selected to fit experimental measurements of the nonlinear transfer curves in the most critical region near saturation. As seen in Fig. 13, they provide a very physical representation of saturation and decline of the AM/AM curve in the region of high input powers (cf. Ref. 26). For the reasons discussed above, however, the details of the nonlinear phase transfer curve were not particularly critical to simulating the dynamics of the experiments incorporating a passband filter in the feedback circuit.

The dimensionless parameters $\delta=2.9, \gamma=1.8$ for the narrowband filter and $\gamma=23.4$ for the wideband one were used in the simulations and were selected on the basis of the experimental setup. Figure 14 shows the wave forms, phase portraits, and spectral plots for the oscillator (3), using parameter values representative of the $40 \mathrm{MHz}$ narrowband 

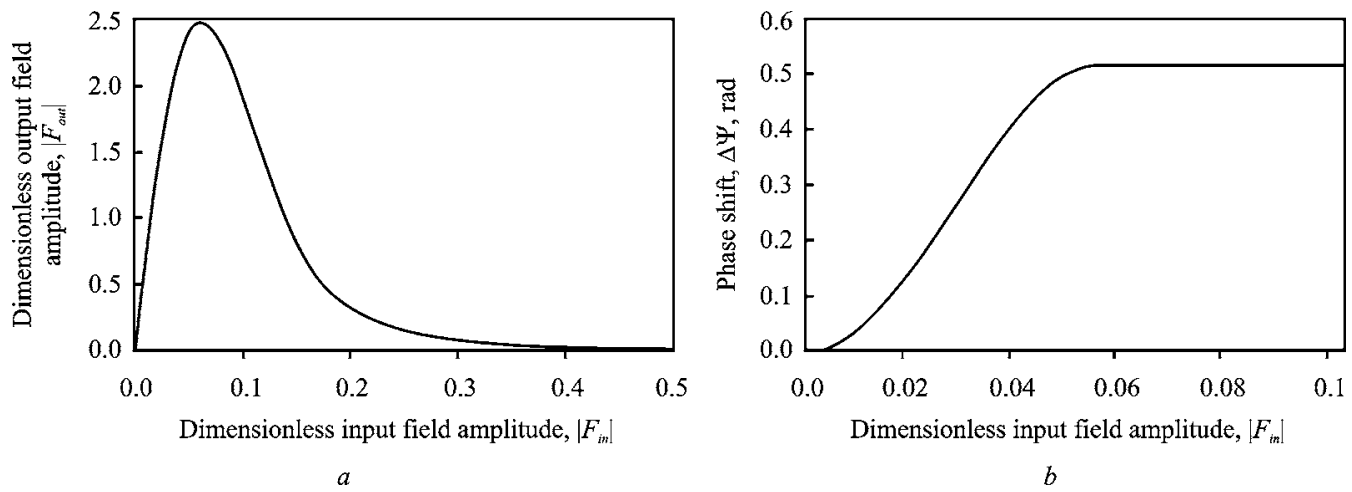

FIG. 13. The analytic approximations of AM/AM (a) and AM/PM (b) curves used in simulation. The dimensionless amplitude (a) and phase shift (b) of the amplified signal are plotted vs the input field amplitude.

feedback filter. The graphs are plotted for different values of feedback attenuation and represent (a) stationary generation, (b) periodic self-modulation, (c) periodic self-modulation after one period-doubling bifurcation, and (d) developed chaos. Additional intermediate steps of period-4 self-modulation and weak Feigenbaum chaos after an infinite sequence of bifurcations are not shown. Note that the time-domain wave forms are all harmonic or quasisinusoidal, in agreement with experimental observations illustrated in Fig. 5.

Illustrative results of the nonlinear TWT oscillator using the wideband $(800 \mathrm{MHz})$ filter model are shown in Fig. 15. These figures also clearly show period doublings and transition to chaos. Note, however, that in agreement with the experimental observations, the self-modulation wave forms are square shaped, not quasisinusoidal. This is the primary observed consequence of using a larger filter bandwidth, for which significantly more ring-loop eigenmodes are allowed to pass through and experience nonlinear positive feedback. Note that in the case of the wideband filter when many modes are present within the filter passband, transient processes are very long owing to mode competition effects.

In Fig. 16 the bifurcation diagram is presented showing alternation of the oscillation regimes with feedback coefficient $\rho$. The diagram was produced by calculating sufficiently long wave forms at discrete values of $\rho$ and by plotting the local wave envelope maxima values. A kink on the curve at $\rho=0.046$ corresponds to self-modulation threshold. One can see a domain of period-doubling transitions to chaos $(\rho \sim 0.075-0.13)$ and a domain of reverse period doublings $(\rho \sim 0.27-0.29)$. Note that only the highest maximum is included in the diagram. In general several local maxima may appear during one period (see, e.g., Fig. 15) leading to a superposition of branches based on different maxima, making the diagram more complicated than one usually observes in a system with period doublings.

In general, this picture agrees quite well with that typical for delayed feedback oscillators (see, for example, Ref. 23) and with the experimental observations. Note that for large values of feedback (large $\rho$ ) there are no chaotic regimes in the simulations. This can be explained by the fact that the AM/AM curve used for these simulations [see Fig. 13(a)] becomes less steep in the area of extremely high input power, i.e., above $\left|F_{\text {in }}\right| \sim 0.2$. This feature would be qualitatively typical of the physical drive curve or AM/AM curve of TWTs, although the experiments conducted for this study did not generally operate at sufficiently high input power as to access this regime.

The results described above confirm that the amplitude nonlinearity plays a primary role in the dynamics. The effects of phase nonlinearity are less pronounced and limited to minor differences. To elucidate the minor role of the phase nonlinearity, we carried out series of simulations with and without the phase nonlinearity. It was revealed that strong phase nonlinearity leads to a somewhat wider and more homogeneous spectrum. Overall, however, the dynamics of this autonomous system were observed to be quite robust to changes of the phase nonlinearity and the generally observed route to chaos through the sequence of period-doubling bifurcations remained intact.

\section{Simulations including an external driving wave}

An important feature of the chaotic behavior in the freerunning oscillator is that the regions of chaos are very narrow, corresponding to a variation of the experiment's feedback attenuator of several $\mathrm{dB}$. This is consistent with the experimental observation that it was difficult to find or sustain chaos in the free-running oscillator configuration using a variable attenuator that was only capable of discrete $1 \mathrm{~dB}$ changes in attenuation. As discussed previously, introducing an external driving wave to the circuit using a commercially synthesized signal generator provided us with a more precise means of dynamic control. It should be noted that an alternative means of introducing more robust chaos would have been to include a delay line into the feedback loop. ${ }^{10}$ Increasing the delay time would lead to a condensation of the eigenmode spectrum, as evident from Eq. (4), which increases the prospects for chaos over larger ranges of control variables. However, a denser eigenmode spectrum also meant that the dynamics would be much more complicated, harder to characterize, and more difficult to understand. Therefore, the option of introducing the driving wave was considered preferable. In addition, this choice also provided a means of introducing an encoded signal, as discussed in Sec. IV.

To model the case of a drive wave applied to the TWT input, Eq. (3) was modified as follows: 

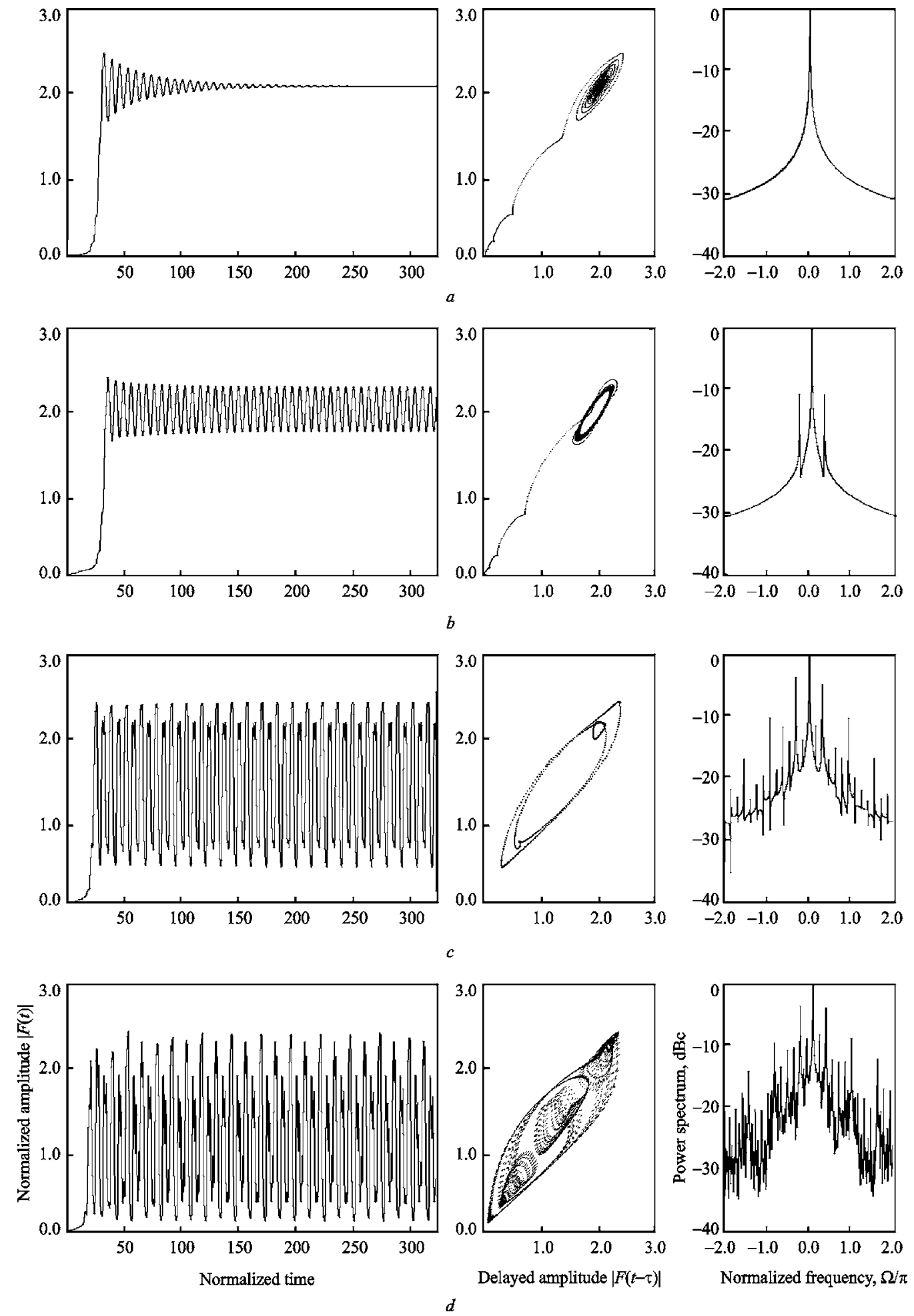

FIG. 14. Wave forms, phase portraits, and power spectra for the narrowband ( $40 \mathrm{MHz})$ filter experiment corresponding to different levels of the feedback coefficient $\rho$. The individual cases correspond to (a) $-26.5 \mathrm{~dB}$, (b) $-26.02 \mathrm{~dB}$, (c) $-21.93 \mathrm{~dB}$, and (d) $-17.0 \mathrm{~dB}$ of feedback attenuation. Wave forms and phase portraits are plotted in dimensionless units. The spectral power densities are normalized to the dominant spectral component (so that $S_{\max }=0 \mathrm{~dB}$ ).

$$
\begin{aligned}
\frac{1}{\gamma} \dot{F}(\tau)+F(\tau)= & \frac{G\left[\left|F^{\prime}(\tau-\delta)\right|\right] F^{\prime}(\tau-\delta)}{\left|F^{\prime}(\tau-\delta)\right|} \\
& \times \exp \left[i \Phi\left(\left|F^{\prime}(\tau-\delta)\right|\right)+\Psi\right],
\end{aligned}
$$

where $F^{\prime}=\rho F+F_{d r}$, and $F_{d r}=F_{0} \exp (i \Omega \tau)$ is the driving signal with amplitude $F_{0}$ and frequency $\Omega$. Applying the driving signal gives a new way to control the system dynamics by varying its power and frequency (relative to the natural eigenmode frequencies).

The application of low driving power generally produced quasiperiodic oscillations. This is consistent with the experimental observations of discrete, multifrequency oscil- 

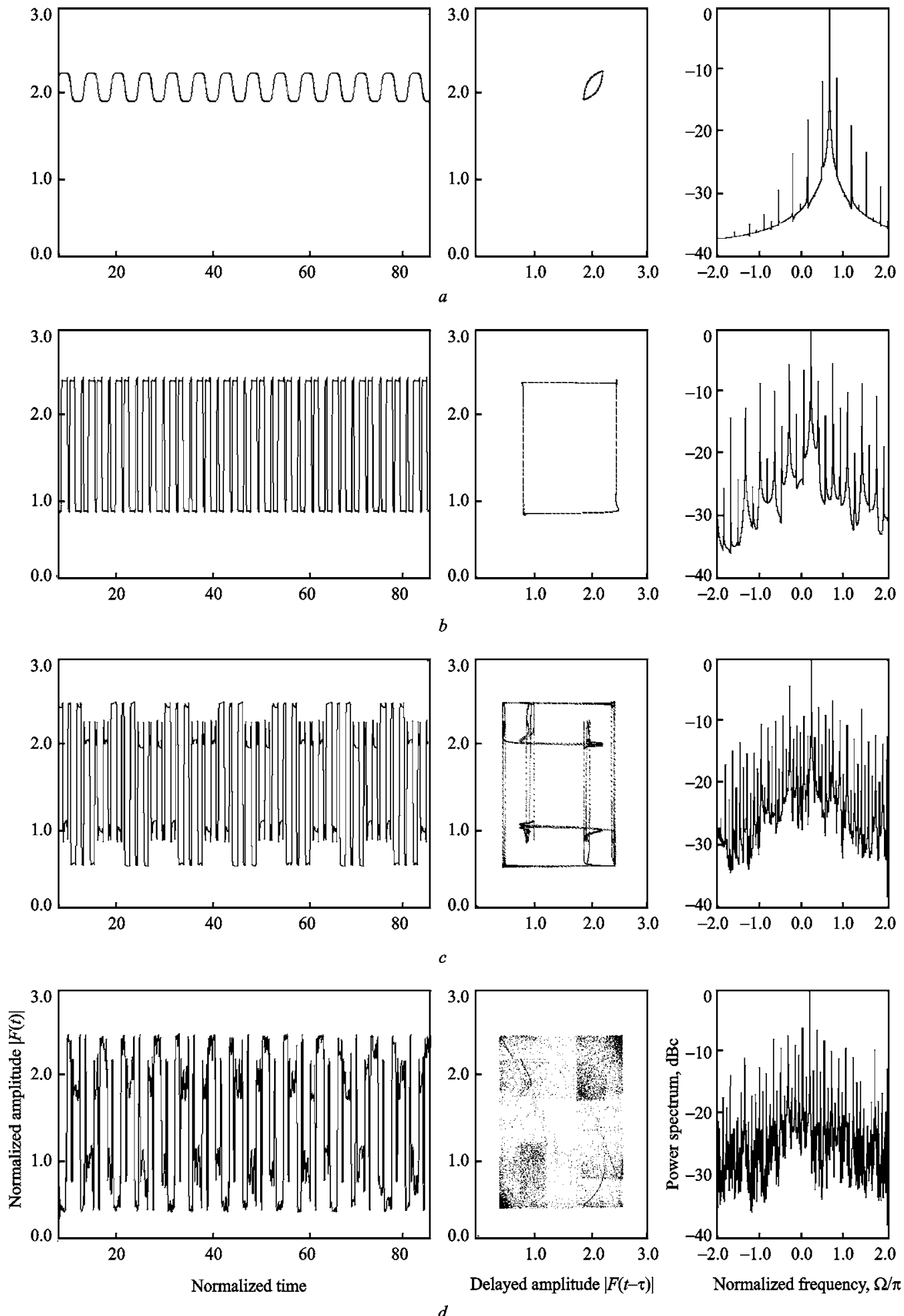

FIG. 15. Wave forms, phase portraits, and power spectra corresponding to different levels of the feedback coefficient $\rho$ for the wider (800 MHz) bandpass feedback filter. The individual cases correspond to (a) $-26.74 \mathrm{~dB}$, (b) $-23.74 \mathrm{~dB}$, (c) $-22.61 \mathrm{~dB}$, and (d) $-21.94 \mathrm{~dB}$ of feedback attenuation.

lations in the "green" regions of Fig. 7. Moderate increase of the driving amplitude led to synchronization regimes based on the driving frequency. Domains of synchronization on the $F_{0}, \Omega$ parameter plane have the form of Arnol'd tongues, similar to those depicted in Fig. 7. Thereafter, varying the relative detuning between the drive frequency and one of the natural oscillation frequencies outside Arnol'd tongues it is possible to observe transitions to chaos.
Principally, two main types of chaos were observed, in conformity to the experiments. The first one-perioddoubling chaos was observed when the drive frequency was approximately halfway in between two natural oscillation frequencies of the system. Figure 17 illustrates a perioddoubling transition while increasing the relative detuning between the drive frequency and the natural oscillation frequencies. In this particular set of simulations, it was elected 


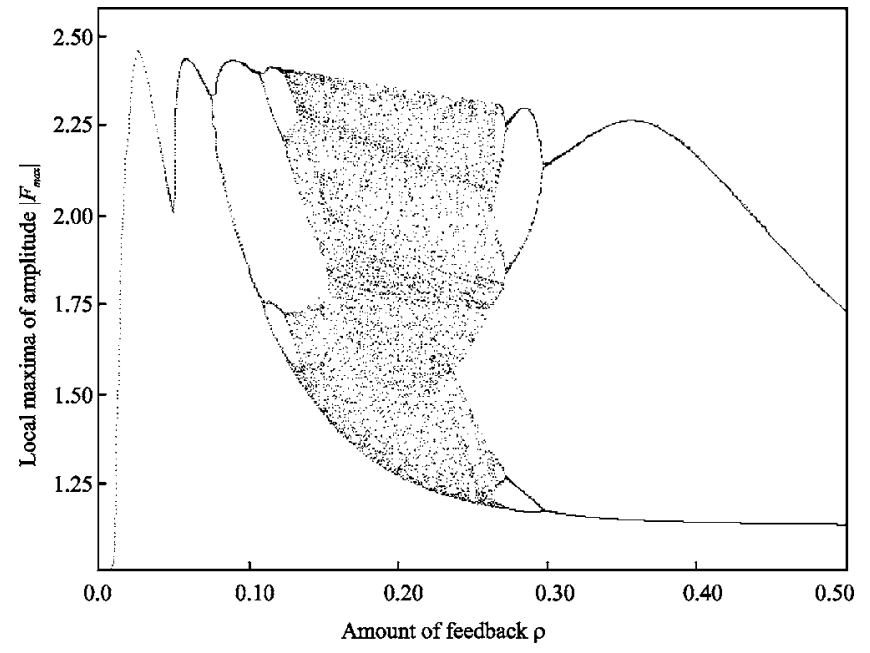

FIG. 16. Bifurcation diagram of the free-running oscillator with the narrowband filter.

to change the drive frequency, keeping the natural oscillation frequencies fixed. Both simulations and experiments verified that the same physics manifested either by varying the drive frequency keeping the feedback circuit constant, or by changing the phase in the feedback leg, keeping the drive frequency constant. The narrow band filter model was used in the simulations. The drive power was set at $8.8 \mathrm{dBm}$. Wave forms, phase portraits, and spectra at different values of dimensionless drive frequency are plotted in Fig. 17. It is apparent that increasing the detuning between the drive frequency and the natural oscillation frequency leads to perioddoubling transition to chaos.

The second type was observed in a close vicinity of synchronization tongues, when the driven frequency was close to the natural oscillator eigenfrequency, thus we named it as loss-of-frequency-locking chaos. Figure 18 displays output signal wave forms and power spectra for that case. For these simulations, the feedback coefficient was set to $\rho=0.1122$ which corresponds to approximately $-19 \mathrm{~dB}$ attenuation in the experiment. These pictures are in a very good agreement with the experimental ones of Fig. 10. At first, the behavior is similar to the classical picture of synchronization via frequency locking [Fig. 18(a)]. When the driving frequency approaches the natural oscillation frequency the period of modulation increases. Note that the output wave forms resemble the experimental wave forms of Fig. 10 (spikes). A very small shift of the driving frequency to a critical value of detuning leads to essentially different oscillations with much greater modulation frequency [Fig. 18(b)]. Note that the onset of this regime occurs via a long and complicated transient process. Next variation of the driving frequency leads to strong chaos [Fig. 18(c)]. This behavior can be attributed to excitation of natural self-modulation sidebands of the freerunning oscillator. Note that frequencies corresponding to those sidebands exist in the experimental spectrum of Fig. 10 (d) rising above the noisy chaos background. Finally, as the frequency is decreased further, we get true frequency locking and there is only one frequency, even though the oscillator has several possible frequencies it could resonate as if left alone [Fig. 18(d)]. These results are, again, consistent with the experimental observations.

More detailed simulations reveal an intriguing selfsimilar "fine structure" of regular and chaotic regimes near the synchronization tongues. The effect is illustrated by Fig. 19 where a bifurcation diagram is plotted versus the driving frequency. This level of detail was not recorded in the experiments, and it is speculated that noise inherent to the experimental system would have masked this fine structure. However, it was possible to conduct the simulations with fairly high resolution and without background noise. Detailed investigation of this fine structure and other features of synchronization and chaos in the driven TWT oscillator is a subject of the ongoing work and will be published elsewhere.

\section{EXPERIMENTAL INVESTIGATION OF SIGNAL ENCODING AND DECODING}

Much investigation has already been conducted on chaotic communications with laser and circuits. This includes both experimental and theoretical investigations in chaotic encoding/decoding for an information transmission scheme as given in Refs. 1, 2, 7, and 8. Recently a direct chaotic communication scheme in microwave band using a delayed feedback klystron oscillator has been considered (see, for example, Ref. 27). These approaches generally require two separate oscillators coupled in a state of synchronization. An alternative scheme introduced by Volkovskii and Rul'kov with nonlinear circuits as given in Ref. 28 and used in Ref. 9 with laser and in Ref. 27 with klystron, respectively, requires only one transmitter oscillator while a receiver contains an amplifier in an open-loop configuration that is driven by the chaotic signal from the transmitter to obtain a synchronous chaotic response.

The Volkovskii-Rul'kov configuration is outlined below. The input signal can be modulated with several different schemes. The basic idea is to establish a chaotic state in the closed-loop oscillator-this will encode $V_{\text {in, }, 1}(t)$. Equations (8) and (9) imply that $V_{\text {out,2}}\left(t+\tau_{d}\right)$ contains the transmitted chaos plus the message signal. A little investigation leads to the results that $V_{\text {out }, 2}\left(t+\tau_{d}\right)-V_{\mathrm{in}, 2}(t)$ equals the message signal as it was input into the system:

$$
\begin{aligned}
& V_{\text {in }, 1}(t)=M(t) \cos \left(\omega_{D} t\right)+V_{\text {out }, 1}\left(t+\tau_{d}\right) \\
& V_{\text {out }, 2}\left(t+\tau_{d}\right)=V_{\text {out }, 1}\left(t+\tau_{d}\right)=V_{\text {in }, 1}(t)-M(t) \cos \left(\omega_{d} t\right) .
\end{aligned}
$$

As part of this study, exploratory experiments into chaos communications were conducted on traveling wave tubes. The Volkovskii-Rul'kov configuration of one closed-loop oscillator and one open-loop receiver was chosen for considerations of being able to match the loop characteristics (bandpass filter, delay time of loop, etc.) with available components and to within reasonable tolerances. Two Varian VTC-6067B1 TWTs were assembled into a circuit as shown in Fig. 20.

Drive power (transfer) curves for the two TWTs, measured at $4.7 \mathrm{GHz}$, are shown in Fig. 21. In the small signal regime, the two curves are almost identical except for a constant difference in small signal gain. Above $5 \mathrm{dBm}$ of drive 

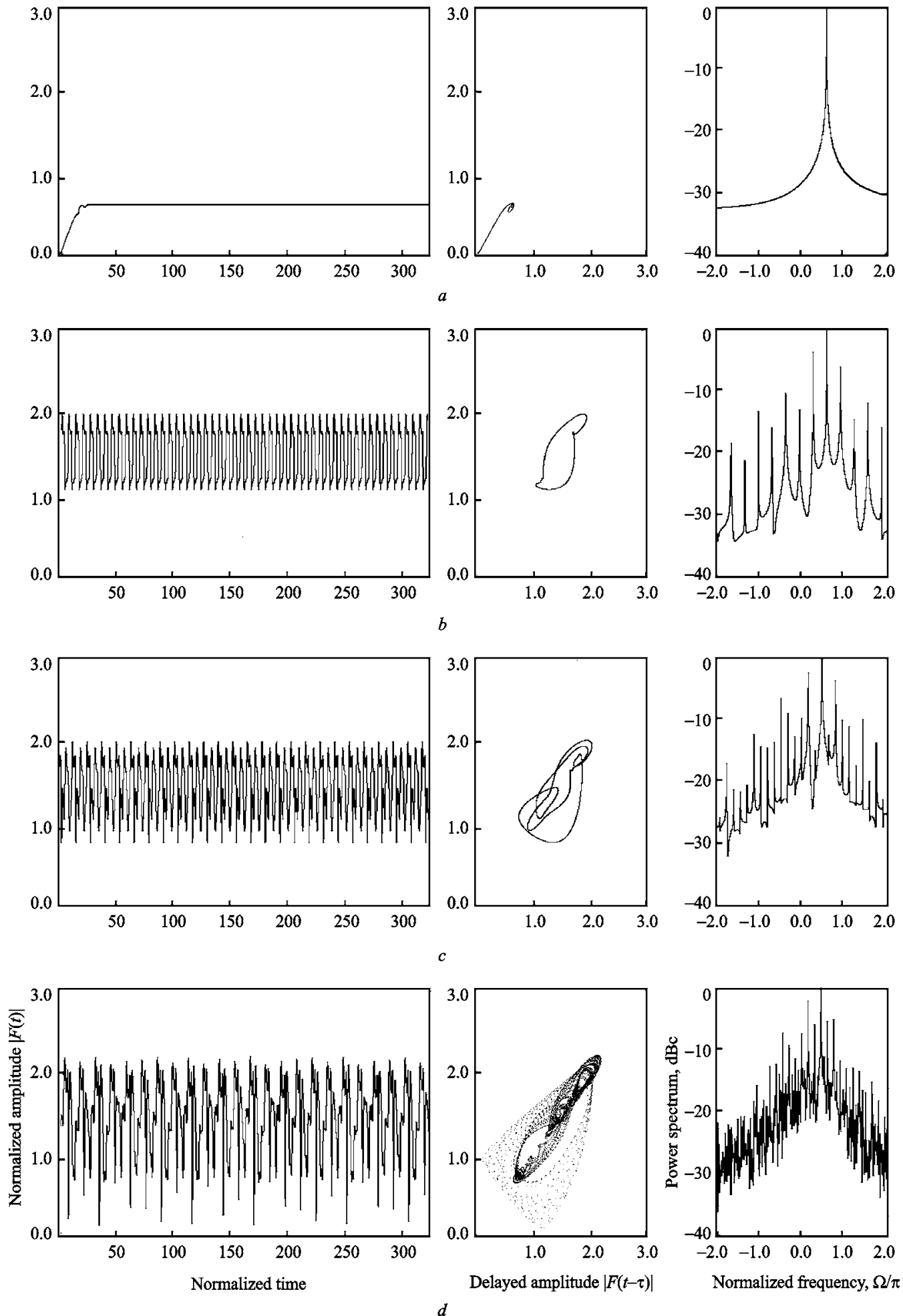

FIG. 17. Wave forms and spectra plotted for the narrow $(40 \mathrm{MHz})$ bandpass feedback filter with external driving amplitude of $8.8 \mathrm{dBm}$. The cases correspond to dimensionless driving frequency $\Omega$ of (a) $0.64 \pi$, (b) $0.55 \pi$, (c) $0.54 \pi$, and (d) $0.45 \pi$. The feedback attenuation was set to $-23.7 \mathrm{~dB}$. The value of feedback phase $\psi$ was equal to $0.45 \pi$.

power, the two curves are identical in the overdriven regime past saturation. The region below the point where the two curves meet (shown by a circle) in Fig. 21 shows the nominal region of chaos operation that corresponded to the experiments described below.

For this initial investigation of signal transmission with a chaotic "carrier," the normally constant amplitude of the drive signal of the chaotic oscillator (the transmitter, for this experiment) was partially amplitude modulated using a $p-i$ $n$-diode modulator, as shown in the top wave form of Fig. 21. The parameters of the chaotic ring-loop transmitter oscillator were adjusted to induce chaos, with the result that the mes- 

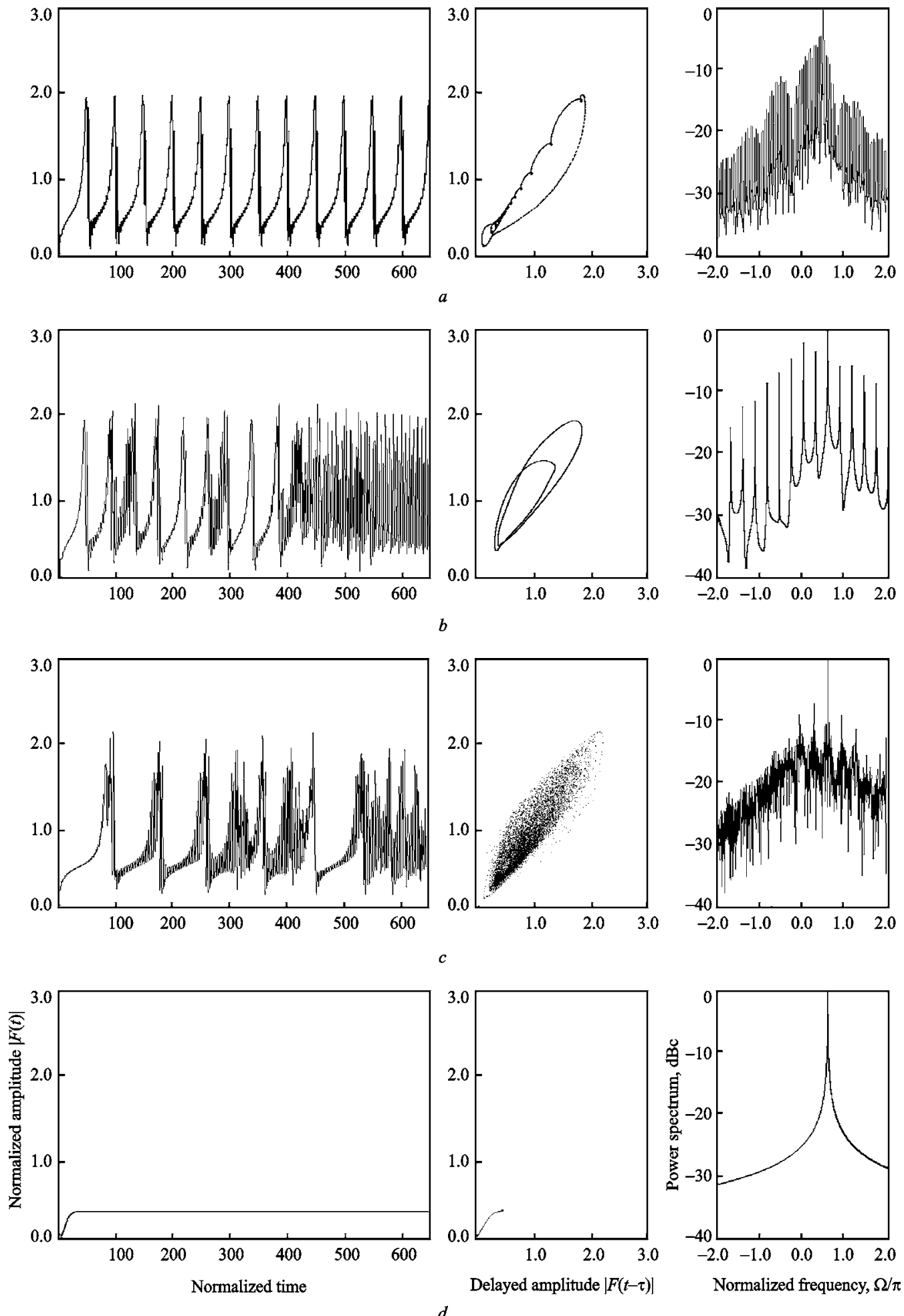

FIG. 18. Wave forms and spectra plotted for the narrow $(40 \mathrm{MHz})$ bandpass feedback filter with external driving amplitude of $11.7 \mathrm{dBm}$. The cases correspond to dimensionless driving frequency $\Omega$ of (a) $0.5 \pi$, (b) $0.505 \pi$, (c) $0.58 \pi$, and (d) $0.6 \pi$. The feedback parameter was set to a value corresponding to an approximate round-trip total attenuation of $-19 \mathrm{~dB}$. The value of feedback phase $\psi$ was equal to $0.65 \pi$.

sage wave form was embedded and masked within the chaos at the position designated by $V_{\text {in, } 1}$ in Fig. 21. The messageembedded-in-chaos wave form is shown as the "TWT1" wave form of Fig. 22. A second TWT was configured in the open-loop configuration, as shown in Fig. 22. The $V_{\text {in, } 1}$ signal was fed to the input of the second TWT through a short coaxial cable. The amplitude of $V_{\mathrm{in}, 2}=V_{\mathrm{in}, 1}$ and $V_{\text {out }, 2}$ were detected with diodes and recorded on a digital oscilloscope. Subsequently, the second TWT's output wave form was numerically time delayed by the estimated round-trip delay time of the experiment's transmitter oscillator feedback path and subtracted from the $V_{\mathrm{in}, 2}$ wave form. The result is dis- 


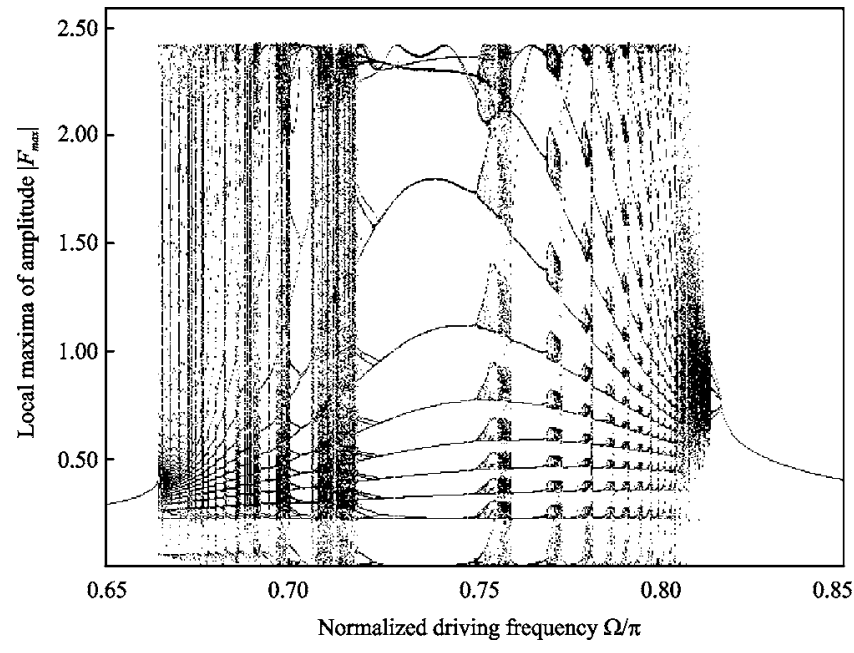

FIG. 19. Bifurcation diagram with the increase of the driving frequency $\Omega$ for the oscillator with the wideband $(800 \mathrm{MHz})$ filter. External driving amplitude is $12 \mathrm{dBm}$, feedback phase $\psi=0.65 \pi$, and the approximate roundtrip total attenuation is $-19 \mathrm{~dB}$. For these simulations, the closest natural oscillation frequency (location of a synchronization tongue) was at $0.66 \pi$.

played as the TWT2 wave form in Fig. 22. The reconstruction of the original message is clearly evident, verifying the feasibility of the technique. Improved results are expected in future experiments wherein the time delay of $V_{\text {out, } 2}$ can be optimized, and an analog subtraction can be also achieved using a high-bandwidth differential amplifier.

\section{SUMMARY AND CONCLUSIONS}

The generation of chaos has been investigated in a driven, delayed-feedback, TWT oscillator. For a free-running oscillator, finding the chaos relies upon precise settings of the feedback attenuation. Addition of a driver signal provided a more robust system in that the dynamics were easily and repeatably controlled by variations in drive signal power and frequency, which were precise by using a commercial signal generator. Dynamics was observed to depend on the feedback bandwidth, achieved here through the use of a feedback filter. Without a filter, robust chaos was observed (less sensitive to small changes in system parameters) but it was extremely wideband $(>10 \mathrm{GHz})$, consistent with the very large bandwidth of positive gain in the experiment's TWT. This regime would be of serious research interest, but was beyond the current abilities to examine experimentally (due to limits on the data acquisition instrumentation bandwidth) and theoretically (due to the very large numbers of simultaneous eigenmodes, along with the large variations in frequency dependence of the nonlinear gain properties of the

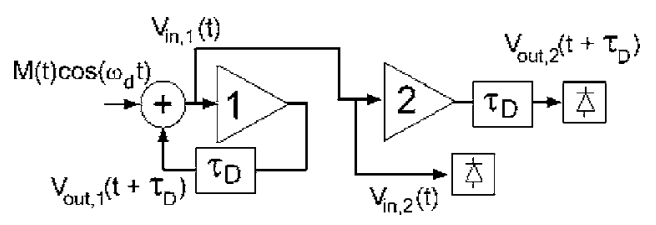

FIG. 20. Volkoskii-Rul'kov configuration. Equations (8) and (9) below correspond to voltages marked in figure.

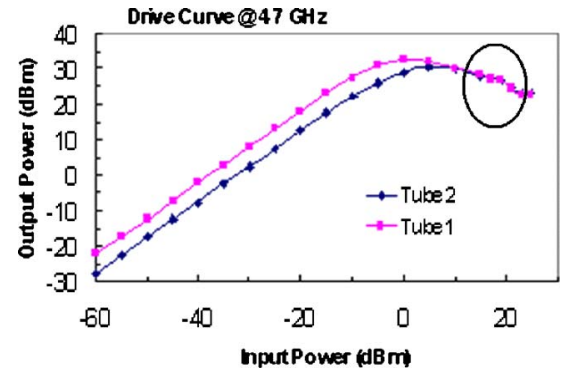

FIG. 21. Transfer curves for the two TWTs used in the chaos communications experiments.

TWT over such a large bandwidth). Consequently, a bandlimiting filter was incorporated into the feedback circuit. Period-doubling transitions to chaos were observed with two different filters of different bandwidths (40 and $800 \mathrm{MHz}$ ), with the exception that the modulation with the narrowband filter was sinusoidal or quasisinusoidal, while it was squaretype modulation with the wider bandwidth filter, as a result of allowing for more natural eigenmodes and thus harmonics of the modulation frequencies in the system. A theoretical model was constructed of the experimental system. Previously published theoretical papers indicated that one effect of the use of the feedback bandpass filter would be to make the amplitude nonlinearity dominant over phase nonlinearity in determining the dynamics of the system. This was also confirmed in preliminary simulations of the model of this paper when a feedback filter was included.

Three regimes of dynamics were experimentally observed with the narrowband filter and the drive signal: discrete multifrequency quasiperiodic oscillation at low drive powers, single-frequency (frequency-locked) oscillation at high drive powers, and chaos near the interface between the other two regimes. Two routes to chaos were observed: period doubling and a new route called loss of frequency locking. The period-doubling type of chaos was generally more robust (less sensitive to small changes in the system parameters). In Arnol'd maps, constructed by varying the drive power and drive frequency, it was observed to exist in islands surrounded by regions of quasiperiodic multifrequency dynamics. Also, the period-doubling chaos was generally observed for drive frequency values approximately halfway in between two natural resonant eigenfrequencies of the freerunning oscillator. In contrast, in the same Arnol'd maps, the loss-of-frequency-locking chaos was observed to exist within thin continuous bands at the edges of the Arnol'd synchronization tongues and in close proximity to one of the natural eigenfrequencies of the free-running oscillator.

In the simulations, the same three dynamical regimes were observed. Moreover, the numerical simulations could identify three types of chaos: the same two as in the experiments, along with a third, quasiperiodic transition to chaos via intermittency. However, this third type, occurring at low drive powers, is more sensitive to the attenuation feedback level, and therefore was not readily studied in the experiments. Also, the numerical simulations indicated that a very detailed fine structure should exist in the bands of loss-offrequency-locking chaos. This was not observed in the ex- 


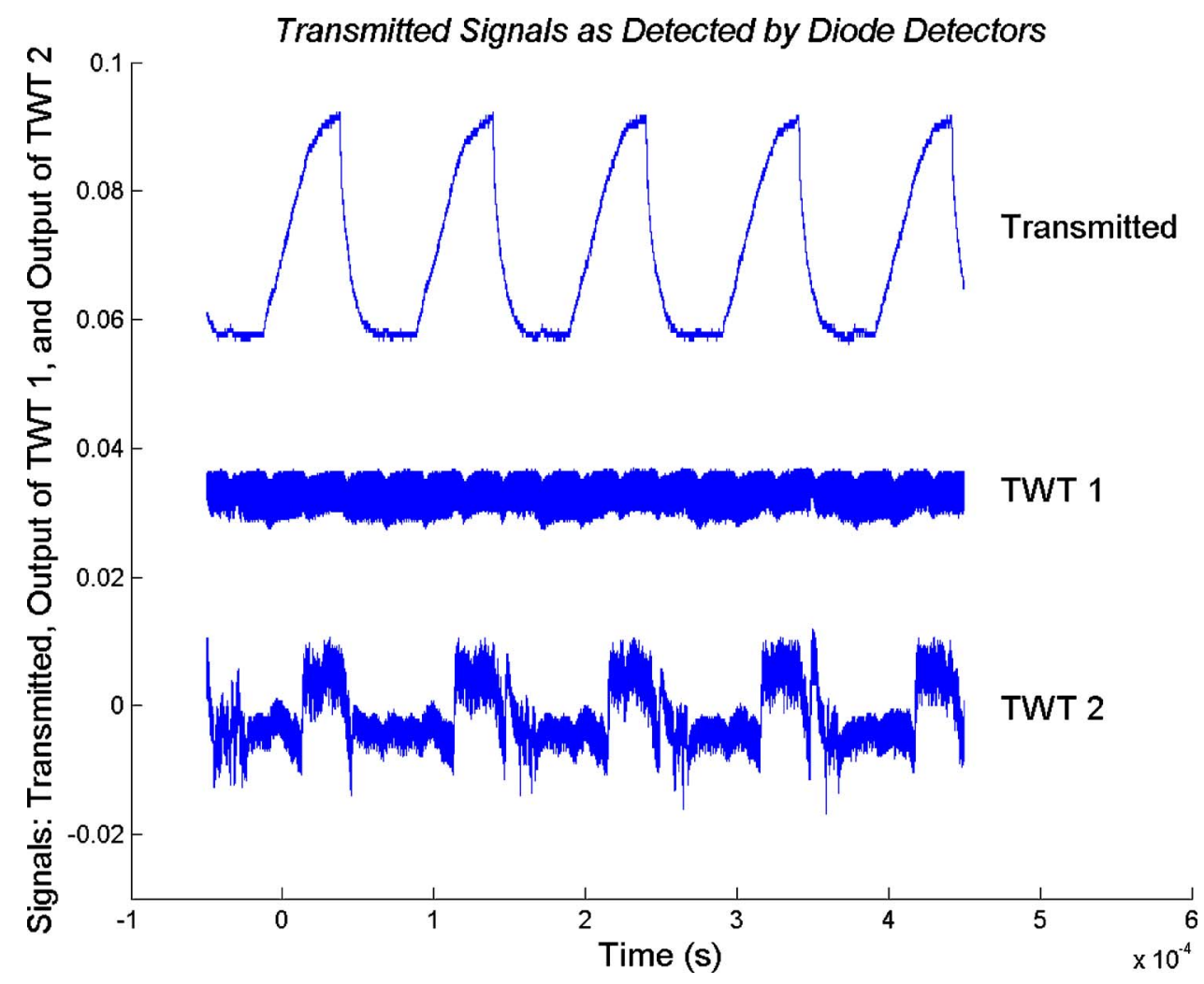

FIG. 22. Illustrative result of chaos communication experiment using two TWTs. The receiver TWT was configured in an open-loop circuit. The signal wave form is labeled "transmitted," the "TWT1" wave form is the signal embedded in chaos, and the approximately reconstructed signal is labeled "TWT2."

periments, either because the level of precision required in the examination would have been much higher than what was available, or, more likely, because this fine structure becomes washed out with the addition of noise, inherent in the experiments. The addition of the noise also facilitates the onset of loss-of-frequency-locking chaos, making it more robust, even though the numerical simulations indicate that it is an inherent response of the system, not requiring noise.

Experiments were conducted on signal chaotic communication between two TWTs, with qualitatively successful results, using an open-loop receiver circuit. Further improvements are expected with a more refined approach using a differential amplifier, noise filtering, and improved signal processing.

\section{ACKNOWLEDGMENTS}

The authors would like to acknowledge many helpful discussions with Dr. T. Antonsen, Dr. G. Nusinovich, Dr. J. Rogers, and Dr. J. C. Sprott. We are thankful to Stephanie Koch and Adam Bush for their help with the chaos communication experiments.

Support for this work is gratefully acknowledged from a grant by the U.S. Air Force Office of Scientific Research, by the U.S. Department of Defense Innovative Microwave Vacuum Electronics Multidisciplinary University Research Initiative (MURI99) grant (managed by U.S. AFOSR), by the University of Wisconsin Vilas Associate faculty research award, by the Russian Federation for Basic Research (Grant No. 05-02-16273), and by the program "Universities of Russia." One of the authors (V.T.) work is also supported by the U.S. Civilian Research and Development Foundation BRHE fellowship for young scientists.
${ }^{1}$ Chaotic Electronics in Telecommunications, edited by M. P. Kennedy, R. Rovatti, and G. Setti (CRC, Boca Raton, FL, 2000).

${ }^{2}$ A. S. Dmitriev and A. I. Panas, Dynamic Chaos: Novel Type of Information Carrier for Communication Systems (Fizmatlit, Moscow, 2002).

${ }^{3}$ A. S. Dmitriev, M. Hasler, A. I. Panas, and K. V. Zakharchenko, Nonlinear Phenom. Complex Syst. 6, 488 (2003).

${ }^{4}$ M. Hasler, Int. J. Bifurcation Chaos Appl. Sci. Eng. 8, 647 (1998).

${ }^{5}$ A. Abel and W. Schwartz, Proc. IEEE 90, 691 (2002).

${ }^{6}$ K. A. Lukin, Proceedings of Noise Radar Technology Workshop (NRTW 2002), edited by K. A. Lukin and W. Miceli (Yalta, Crimea, Ukraine, 2002), p. 13.

${ }^{7}$ I. Fischer, Y. Liu, and P. Davis, Phys. Rev. A 62, 011801(R) (2000).

${ }^{8}$ J. Paul, S. Sivaprakasam, P. S. Spencer, P. Rees, and K. A. Shore, Electron. Lett. 38, 28 (2002).

${ }^{9}$ G. D. VanWiggeren and R. Roy, Science 279, 1198 (1998).

${ }^{10}$ V. A. Katz, Radiophys. Quantum Electron. 28, 161 (1985).

${ }^{11}$ V. Ya. Kislov, E. A. Myasin, and N. N. Zalogin, Radiotekh. Elektron. (Moscow) 25, 2160 (1980).

${ }^{12}$ Yu. V. Anisimova, A. S. Dmitriev, N. N. Zalogin, V. I. Kalinin, V. Ya. Kislov, and A. I. Panas, JETP Lett. 37, 458 (1983).

${ }^{13}$ V. Dronov, M. R. Hendrey, T. M. Antonsen, and E. Ott, Chaos 14, 30 (2004).

${ }^{14}$ N. S. Ginzburg, S. P. Kuznetsov, and T. N. Fedoseeva, Izv. Vyssh. Uchebn. Zaved., Radiofiz. 21, 1037 (1978) [Sov. Radiophys. Electron. 21, 728 (1978)].

${ }^{15}$ Yu. P. Bliokh, A. V. Borodkin, M. G. Liubarskii, I. N. Onischenko, and Ya. B. Fainberg, Appl. Nonlin. Dynamics 1, 34 (1993).

${ }^{16}$ Yu. P. Bliokh, M. G. Liubarskii, V. O. Podobinskii, and Ya. B. Fainberg, Fiz. Plazmy 20, 718 (1994) [Plasma Phys. Rep. 20, 648 (1994)].

${ }^{17}$ Yu. P. Bliokh, M. G. Liubarskii, V. O. Podobinskii, Ya. B. Fainberg, G. S. Nusinovich, S. Kobayashi, Y. Carmel, and V. L. Granatstein, Phys. Plasmas 5, 4061 (1998).

${ }^{18}$ N. M. Ryskin, Radiophys. Quantum Electron. 47, 116 (2004).

${ }^{19}$ N. M. Ryskin, V. N. Titov, S. T. Han, J. K. So, K. H. Jang, Y. B. Kang, and G. S. Park, Phys. Plasmas 11, 1194 (2004).

${ }^{20}$ B. Goplen, L. Ludeking, D. Smithe, and G. Warren, Comput. Phys. Commun. 87, 54 (1995).

${ }^{21}$ T. Weiland, Part. Accel. 17, 227 (1985).

${ }^{22}$ S. Bhattacharjee, J. H. Booske, C. L. Kory, D. W. van der Weide, W.-J. Lee, S. Limbach, S. Gallagher, J. D. Welter, M. R. Lopez, R. M. Gilgenbach, R. L. Ives, M. E. Read, R. Divan, and D. C. Mancini, IEEE 
Trans. Plasma Sci. 32, 1002 (2004)

${ }^{23}$ N. M. Ryskin and A. M. Shigaev, Zh. Tekh. Fiz. 72 (8), 1 (2002) [Tech. Phys. 47, 795 (2002)].

${ }^{24}$ S. P. Kuznetsov, Izv. Vyssh. Uchebn. Zaved., Radiofiz. 25, 1410 (1982).
${ }^{25}$ T. M. Antonsen and B. Levush, Phys. Fluids B 1, 1097 (1989).

${ }^{26}$ V. Ya. Kislov, Radiotekh. Elektron. (Moscow) 25, 1683 (1980).

${ }^{27}$ A. M. Shigaev, B. S. Dmitriev, Y. D. Zharkov, and N. M. Ryskin, IEEE Trans. Electron Devices 52, 790 (2005).

${ }^{28}$ A. R. Volkovskii and N. F. Rul'kov, Tech. Phys. Lett. 19, 97 (1993). 